\title{
Force Estimation and Prediction from Time-Varying Density Images
}

\author{
Srinivasan Jagannathan, Berthold Klaus Paul Horn, Purnima Ratilal, and \\ Nicholas Constantine Makris
}

\begin{abstract}
We present methods for estimating forces which drive motion observed in density image sequences. Using these forces, we also present methods for predicting velocity and density evolution. To do this, we formulate and apply a Minimum Energy Flow (MEF) method which is capable of estimating both incompressible and compressible flows from time-varying density images. Both the MEF and force-estimation techniques are applied to experimentally obtained density images, spanning spatial scales from micrometers to several kilometers. Using density image sequences describing cell splitting, for example, we show that cell division is driven by gradients in apparent pressure within a cell. Using density image sequences of fish shoals, we also quantify 1) intershoal dynamics such as coalescence of fish groups over tens of kilometers, 2) fish mass flow between different parts of a large shoal, and 3) the stresses acting on large fish shoals.
\end{abstract}

Index Terms-Force estimation, density prediction, compressible flow estimation, minimum energy flow.

\section{INTRODUCTION}

$\mathrm{E}$ STIMATING velocity and force fields from image sequences is an essential and often first step of analysis in a wide variety of applications such as object detection and tracking, robot navigation, visual odometry, medical imaging, remote sensing, and satellite imagery. Image sequences used in these applications describe both compressible and incompressible flows. A variety of methods exist for estimating velocity fields, such as Optical Flow [23] and pressure gradients [38], [54] from time-varying images describing incompressible motion.

In this paper, we develop and apply methods for estimating the forces driving motion observed in density image sequences, where pixel values can be modeled as proportional to the density of a compressible fluid. Using these forces, we also present methods for predicting future velocity and density values. To do this, we formulate and apply a Minimum Energy Flow (MEF) method to estimate velocity fields from image sequences, describing both compressible and incompressible flows.

The MEF and force-estimation techniques can be generally applied to any density image sequence, where pixel values can be modeled as proportional to the density of a compressible fluid. Here, for example, we demonstrate these

- S. Jagannathan and N.C. Makris are with the Department of Mechanical Engineering, Massachusetts Institute of Technology, 77 Massachusetts Avenue, Cambridge, MA 02139. E-mail: \{jsrini, makris\}@mit.edu.

- B.K.P. Horn is with the Computer Science and Artificial Intelligence Laboratory, Department of Electrical Engineering and Computer Science, Massachusetts Institute of Technology, Room 32-D434, 32 Vasar Street, Cambridge, MA 02139. E-mail: bkph@csail.mit.edu.

- P. Ratilal is with the Department of Electrical and Computer Engineering, Northeastern University, Room 311, 302 Stearns Center, 360 Huntington Avenue, Boston, MA 02115. E-mail: purnima@ece.neu.edu.

Manuscript received 8 Apr. 2010; revised 26 July 2010; accepted 28 July 2010; published online 30 Sept. 2010.

Recommended for acceptance by S. Belongie.

For information on obtaining reprints of this article, please send e-mail to: tpami@computer.org, and reference IEEECS Log Number

TPAMI-2010-04-0251.

Digital Object Identifier no. 10.1109/TPAMI.2010.185. techniques at the microscale by quantifying the dynamics of cell division, and at the macroscale by quantifying fish shoal dynamics over tens of kilometers. Using density images of a cell undergoing mitosis [19], we quantify the velocity, net force, and apparent pressure fields inside the cell. We find that the cell division is driven by the formation of two regions of low apparent pressure at opposite sides of the cell and a region of high apparent pressure at the center. Using fish population density images obtained with an Ocean Acoustic Waveguide Remote Sensing (OAWRS) [32], [27] system, we quantify 1) intershoal dynamics such as coalescence of fish groups over tens of kilometers, 2) fish mass flow between different parts of a large shoal, and 3) the stresses acting on large fish shoals. To study collective behavior, large animal groups, including fish shoals, are often modeled as compressible fluids [51], [52]. Such theoretical group behavior models predict average velocities and forces inside animal groups, which can be verified using our MEF and force estimation techniques.

\section{BACKGROUND}

Classical motion estimation from image sequences describing incompressible motion is based on Horn and Schunk's [23] work on determining Optical Flow. Barron et al. [4] review and compare the different optical flow techniques, including [23], [31], [53], [37], [2], [47], [22], [55], and [18], where the $2 \mathrm{D}$ velocity field $(\mathbf{u})$ is computed from spatial and temporal variations in the image intensity $(E)$ patterns by minimizing a global cost function of the form

$$
\iint_{\Omega} f\left(\frac{\partial E}{\partial t}+\nabla E \cdot \mathbf{u}\right)+\lambda g(|\nabla \mathbf{u}|) \mathrm{d} x \mathrm{~d} y,
$$

where $f(\cdot)$ and $g(\cdot)$ are monotonically increasing functions (usually the magnitude squared of the argument), $\lambda$ is an empirically determined weight, and $\Omega$ is the image plane.

The above choice of cost function is especially suited for incompressible motion estimation since 1) the argument of 
$f(\cdot)$ should be zero in an incompressible fluid [5] when $E$ is proportional to the density $\rho$ of the fluid, and 2) minimizing $g(\cdot)$, also known as the "unsmoothness of flow" criterion, suppresses large gradients in velocity which are usually associated with compressible flows.

In compressible flow estimation, a modification of the Optical Flow technique is to replace the first term in the cost function with the corresponding term from the compressible equation of continuity [5] for fluids. Methods based on this modification [1], [7], [56], however, retain the "unsmoothness of flow" criterion, which may not be suitable for estimating flows with large spatial gradients in the velocity field, as we show in comparisons with the MEF approach (Appendix A). In the case of compressible flows, it is the spatial gradients in velocity which contain information about the compressible nature of the motion, and using the "unsmoothness of flow" criterion may distort the velocity field [13]. Higher order penalty functions such as "second order div-curl" minimization [49] have been suggested for fluid flow estimation. These methods penalize sharp changes in vorticity and divergence of flow, which may not be appropriate in estimating general turbulent flow either.

Penalty functions other than the "unsmoothness of flow" of Optical Flow have also been proposed for nonrigid deformation estimation. Devalminck and Dubus [13], and others [35], [41], [50] propose formulations based on minimizing the strain energy of deformation, which is applicable only for objects that undergo elastic deformations with a known stress-strain relationship but not for fluids undergoing compressible motion.

The MEF technique uses a physically motivated penalty function that does not directly depend on the spatial gradients of velocity. The total kinetic energy is used instead of the "unsmoothness of flow" criterion. The choice of kinetic energy is motivated by the Least Action Principle [34], according to which the evolution of a physical system from one state to another corresponds to the minimum of the action [29]. Since we are interested in estimating compressible fluid flow, this principle reduces to minimizing the kinetic energy of fluid particles corresponding to the density at an image pixel.

Our force-estimation technique uses the flow fields computed by MEF as inputs and is applicable to both steady and unsteady flows. That is, the forces are estimated by taking into account temporal fluctuations in the velocity field. The nonlinear Navier-Stokes equation [5] is used and both conservative and nonconservative forcing terms are assumed to be present. The force-estimation technique itself is a separate "module" that can, in general, have inputs from any motion estimation model. We have developed and applied a MEF technique for motion esimation because our method performs better than existing techniques of compressible flow estimation (Appendix A).

\section{FORMULATION}

\subsection{Velocity Field}

Let $\rho(x, y, t)$ be the density corresponding to a point $(x, y)$ in the image plane $\Omega$ at time $t$. If we assume that $\rho$ is the density of a compressible fluid, then in the absence of any sources and sinks, the velocities are constrained by the equation of continuity [5]

$$
\frac{\partial \rho}{\partial t}+\frac{\partial}{\partial x}(\rho u)+\frac{\partial}{\partial y}(\rho v)=0
$$

where $u$ and $v$ are the components of the flow velocity in the $x$ and $y$ directions, respectively.

This is a single equation relating the measured spatial and temporal variations of density and the two unknown velocity components $u$ and $v$. To determine a particular velocity field, we set up an optimization problem where we take the square of the error in the constraint (the left side of (1)) and add a multiple of the kinetic energy of the system

$$
T=\rho\left(u^{2}+v^{2}\right)
$$

as a penalty term or objective function, and minimize the following integral over $\Omega$ :

$$
\iint_{\Omega}\left[\left(\frac{\partial \rho}{\partial t}+\frac{\partial(\rho u)}{\partial x}+\frac{\partial(\rho v)}{\partial y}\right)^{2}+\lambda \rho\left(u^{2}+v^{2}\right)\right] \mathrm{d} x \mathrm{~d} y .
$$

The velocity field we determine through this minimization is the one that results in the least kinetic energy while making the deviation from satisfying the continuity equation as small as possible.

The term $\lambda$ is a constant that defines the "penalty for" high kinetic energy in the solution. We expect that large values of $\lambda$ will tend to suppress high kinetic energy excursions in the solution (at the cost of not matching the constraint equation as well), while small values of $\lambda$ will tend to make the solution match the constraint equation more closely (at the cost of being more sensitive to measurement noise).

For convenience, we now define

$$
\bar{u}=\rho u \quad \text { and } \quad \bar{v}=\rho v
$$

representing the mass flow rates in the $x$ and $y$ directions, respectively. We can rewrite (3) in terms of these flow rates as

$$
\begin{gathered}
\iint_{\Omega}\left(\rho_{t}+\bar{u}_{x}+\bar{v}_{y}\right)^{2}+\frac{\lambda}{\rho}\left(\bar{u}^{2}+\bar{v}^{2}\right) \mathrm{d} x \mathrm{~d} y \\
=\iint_{\Omega} F\left(\bar{u}, \bar{u}_{x}, \bar{u}_{y}, \bar{v}, \bar{v}_{x}, \bar{v}_{y}\right) \mathrm{d} x \mathrm{~d} y,
\end{gathered}
$$

where the subscripts indicate the variable with respect to which partial derivatives are to be taken. Minimization of (5) can be treated as a problem of the calculus of variations, where we solve the following set of Euler-Lagrange equations:

$$
\begin{aligned}
& F_{\bar{u}}-\frac{\partial}{\partial x} F_{\bar{u}_{x}}-\frac{\partial}{\partial y} F_{\bar{u}_{y}}=0, \\
& F_{\bar{v}}-\frac{\partial}{\partial x} F_{\bar{v}_{x}}-\frac{\partial}{\partial y} F_{\bar{v}_{y}}=0 .
\end{aligned}
$$

Substituting the expression for $F$ into (6) and (7) leads to

$$
\begin{aligned}
& \bar{u}=\frac{\rho}{\lambda}\left(\rho_{t x}+\bar{u}_{x x}+\bar{v}_{x y}\right), \\
& \bar{v}=\frac{\rho}{\lambda}\left(\rho_{t y}+\bar{u}_{x y}+\bar{v}_{y y}\right) .
\end{aligned}
$$


In Appendix B, we present a numerical technique to solve (8) and (9).

Earlier work by Fitzpatrick [17] involves a strict enforcement of the continuity constraint, which may not hold in the presence of measurement noise. A Lagrangian multiplier, denoted by $\lambda(x, y)$, is used as a spatially varying unknown, and closed form analytic solutions are pursued. In the formulation here, departures from satisfying the continuity condition are allowed, but penalized. Additionally, we have used a fixed multiplier $\lambda$ to weigh the energy term. We have assumed that the changes in pixel intensity in the image sequences are purely due to the motion of objects imaged and not due to the motion of the observer. It is possible to correct for observer motion prior to applying MEF. The computational techniques presented in the paper work well for imaging applications with high frame rates. For low frame-rate applications, a coarse-to-fine approach as described in [6], [15] may be employed.

\subsection{Force Field}

A velocity field can be the result of an underlying force field driving the motion. We can determine these forces using the Navier-Stokes equation [5] for compressible flow in two dimensions:

$$
\rho\left(\frac{\partial \mathbf{U}}{\partial t}+(\mathbf{U} \cdot \nabla) \mathbf{U}\right)=-\nabla p+\mathbf{F}
$$

where $\mathbf{U}=(u, v)$ is the vector velocity field, $p$ is the pressure field, and $\mathbf{F}=\left(f_{1}, f_{2}\right)$ is any external "force density" (body force per unit volume) acting on the fluid. The right-hand side of (10) is the sum of a conservative force per unit volume $(\nabla p)$ and a nonconservative force per unit volume $(\mathbf{F})$. The $x$ and $y$ components, respectively, of this vector equation are

$$
\begin{gathered}
\rho\left(u_{t}+u u_{x}+v u_{y}\right)=-p_{x}+f_{1}, \\
\rho\left(v_{t}+u v_{x}+v v_{y}\right)=-p_{y}+f_{2},
\end{gathered}
$$

where subscripts again indicate the variable with respect to which partial derivatives are to be taken. For special cases of fluid flow when either the conservative force or the nonconservative force is zero, the system of (11) and (12) directly provide us the solution for either $\left(f_{1}, f_{2}\right)$ or $p$. In the more general case that we consider here, we assume that neither $\nabla p$ nor $\left(f_{1}, f_{2}\right)$ terms can be neglected and are comparable to each other.

Determining the unknowns $p, f_{1}$, and $f_{2}$ from (11) and (12) is an ill-posed problem, which we will reframe as two decoupled variational problems in order to determine approximate least-squares solutions.

Subtraction of the $y$ derivative of (11) and the $x$ derivative of (12) eliminates $p$ and yields

$$
\begin{aligned}
& \frac{\partial}{\partial y}\left[\rho\left(u_{t}+u u_{x}+v u_{y}\right)\right]-\frac{\partial}{\partial x}\left[\rho\left(v_{t}+u v_{x}+v v_{y}\right)\right. \\
& \quad=\frac{\partial f_{1}}{\partial y}-\frac{\partial f_{2}}{\partial x} .
\end{aligned}
$$

We then find $\left(f_{1}, f_{2}\right)$ that minimizes

$$
\begin{aligned}
& \iint_{\Omega} \| \frac{\partial}{\partial y}\left[\rho\left(u_{t}+u u_{x}+v u_{y}\right)\right]-\frac{\partial}{\partial x}\left[\rho\left(v_{t}+u v_{x}+v v_{y}\right)\right] \\
& -\left(\frac{\partial f_{1}}{\partial y}-\frac{\partial f_{2}}{\partial x}\right) \|^{2} \mathrm{~d} x \mathrm{~d} y .
\end{aligned}
$$

The solutions $f_{1}, f_{2}$ are then given by the following EulerLagrange equations:

$$
\begin{aligned}
\frac{\partial^{2} f_{1}}{\partial y^{2}}= & \frac{\partial^{2}}{\partial y^{2}}\left[\rho\left(u_{t}+u u_{x}+v u_{y}\right)\right] \\
& -\frac{\partial^{2}}{\partial x \partial y}\left[\rho\left(v_{t}+u v_{x}+v v_{y}\right)\right]+\frac{\partial^{2} f_{2}}{\partial x \partial y} \\
\frac{\partial^{2} f_{2}}{\partial x^{2}}= & \frac{\partial^{2}}{\partial x \partial y}\left[\rho\left(u_{t}+u u_{x}+v u_{y}\right)\right] \\
& -\frac{\partial^{2}}{\partial x^{2}}\left[\rho\left(v_{t}+u v_{x}+v v_{y}\right)\right]-\frac{\partial^{2} f_{1}}{\partial x \partial y} .
\end{aligned}
$$

The coupled equations (14) and (15) are solved using a fixed-point iteration technique, which is described in Appendix C.

After determining $\left(f_{1}, f_{2}\right)$, we again use (11) and (12) to solve for $p$

$$
\begin{gathered}
p_{x}=-\rho\left(u_{t}+u u_{x}+v u_{y}\right)+f_{1}, \\
p_{y}=-\rho\left(v_{t}+u v_{x}+v v_{y}\right)+f_{2} .
\end{gathered}
$$

This is a Dirichlet Boundary Value Problem and, in general, is overconstrained. For example, in the computation domain $(x \in[0, L], y \in[0, L])$, the explicit integration of (16) yields

$$
p(x, y)=\int_{0}^{x}\left[-\rho\left(u_{t}+u u_{x}+v u_{y}\right)+f_{1}\right] \mathrm{d} x+p(0, y),
$$

which may not satisfy the boundary condition at $x=L$.

In order to obtain a best fit solution for the system of (16) and (17), we reframe it as a variational problem. One way to do this is to find the solution $p$ that minimizes the square of the euclidean norm of the residues of (16) and (17), much like the procedure adopted to find $\left(f_{1}, f_{2}\right)$. We thus minimize

$$
\begin{aligned}
& \iint_{\Omega}\left[p_{x}+\rho\left(u_{t}+u u_{x}+v u_{y}\right)-f_{1}\right]^{2} \\
& \quad+\left[p_{y}+\rho\left(v_{t}+u v_{x}+v v_{y}\right)-f_{2}\right]^{2} \mathrm{~d} x \mathrm{~d} y .
\end{aligned}
$$

The Euler-Lagrange equation for this variational problem is

$$
\begin{aligned}
\nabla^{2} p= & \frac{\partial}{\partial x}\left[-\rho\left(u_{t}+u u_{x}+v u_{y}\right)\right]+\frac{\partial f_{1}}{\partial x} \\
& +\frac{\partial}{\partial y}\left[-\rho\left(v_{t}+u v_{x}+v v_{y}\right)\right]+\frac{\partial f_{2}}{\partial y},
\end{aligned}
$$

where $\nabla^{2}$ is the Laplacian. We solve this inhomogeneous Laplace equation using a fixed-point iteration, in Appendix C.

\subsection{Predicting Densities Using Forces}

The ability to quantify forces also provides us with a method to predict future density distributions once we have an initial estimate of the velocity field and the force field. 


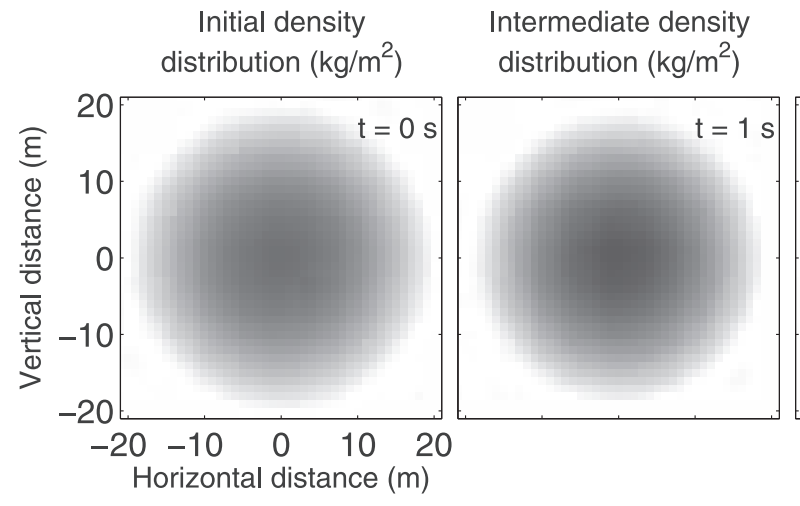

(a)

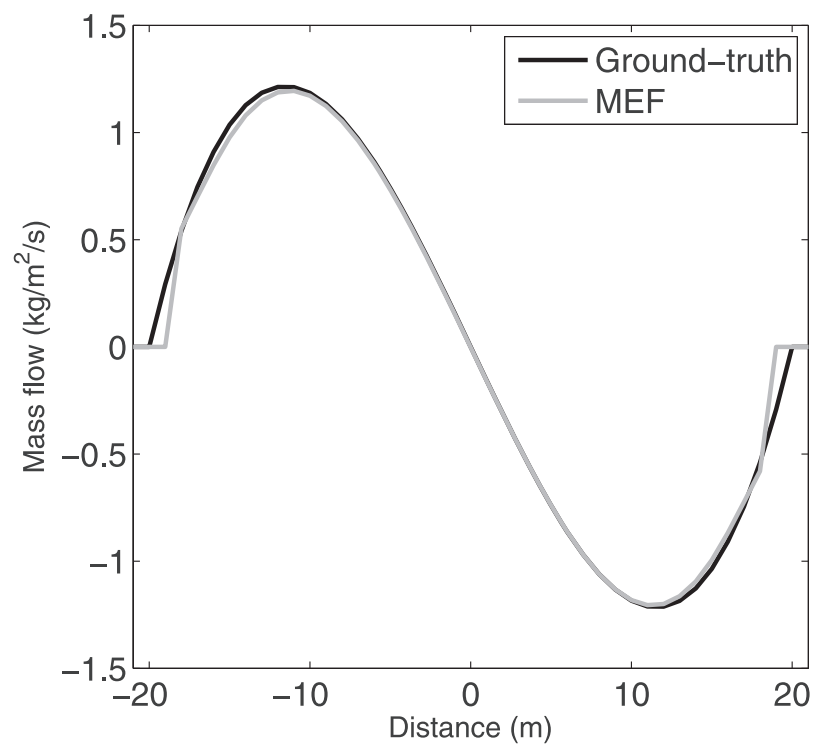

(e)
Final density distribution $\left(\mathrm{kg} / \mathrm{m}^{2}\right)$

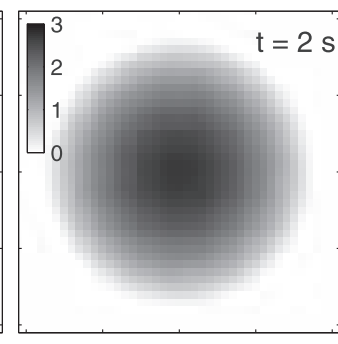

(c)
Pressure distribution $\left(\mathrm{kg} / \mathrm{m} / \mathrm{s}^{2}\right)$

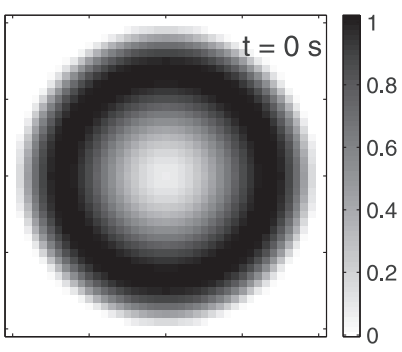

(d)

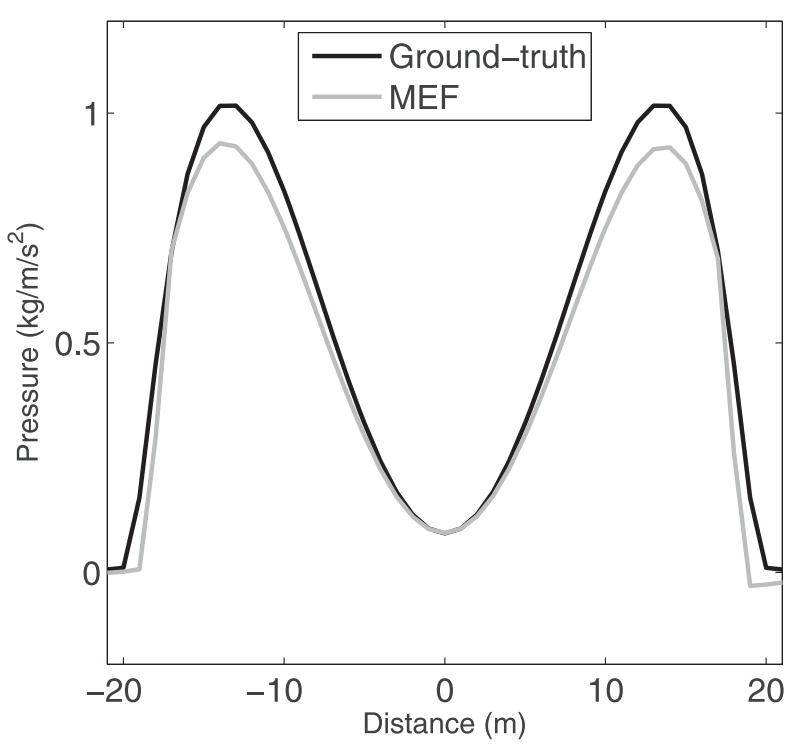

(f)

Fig. 1. (a) Initial, (b) intermediate, and (c) final density distributions of a contracting density feature. (d) The ground-truth pressure distribution that results in contraction. (e) Comparison between ground-truth and MEF-computed horizontal mass flow rates at $\mathrm{t}=0 \mathrm{~s}$ along the $y=0$ cut in (a). (f) Comparison between ground-truth and MEF-computed pressures at $\mathrm{t}=0 \mathrm{~s}$ along the $y=0$ cut in (a). The maximum error in flow estimates is less than 5 percent, while the maximum error in the pressure estimate is $\sim 10$ percent.

In order to do this, we assume that the initial force computed stays constant for some time before there is a substantial change in its magnitude and spatial distribution. This means that over some time scale, the accelerations (or the driving forces) remain constant. Under these assumptions, we suggest the following prediction scheme:

\section{- $\quad$ Step 1}

Obtain density data $\rho^{(n)}, \rho^{(n+1)}, \rho^{(n+2)}$

(superscripts indicate time steps).

- $\quad$ Step 2

Compute $\left(u^{(n)}, v^{(n)}\right)$ and $\left(u^{(n+1)}, v^{(n+1)}\right)$ using $\rho^{(n)}$, $\rho^{(n+1)}, \rho^{(n+2)}$, and (8) and (9).

- $\quad$ Step 3

- $\quad$ Step 4

Calculate $\nabla p^{(n)}$ and $\mathbf{F}^{(n)}$ using (14), (15), and (19).

Set

$$
\begin{aligned}
\nabla p^{(n+1)} & \leftarrow \nabla p^{(n)}, \\
\mathbf{F}^{(n+1)} & \leftarrow \mathbf{F}^{(n)} .
\end{aligned}
$$

- $\quad$ Step 5

$$
\text { Use }\left(u^{(n+1)}, v^{(n+1)}\right), \rho^{(n+1)} \text { in (11) and (12) and }
$$
compute $\left(u^{(n+2)}, v^{(n+2)}\right)$.

- Step 6

- $\quad$ Step 7

$$
\text { Use }\left(u^{(n+2)}, v^{(n+2)}\right) \text { and } \rho^{(n+1)} \text { in (1) to predict } \rho^{(n+3)} \text {. }
$$

Repeat steps 1-6 by setting

$$
\begin{aligned}
\rho^{(n)} & \leftarrow \rho^{(n+1)}, \\
\rho^{(n+1)} & \leftarrow \rho^{(n+2)}, \\
\rho^{(n+2)} & \leftarrow \rho^{(n+3)} .
\end{aligned}
$$

\section{Applications}

\subsection{Synthetic Image Sequences}

To evaluate the performance of the MEF method, we use synthetic image sequences describing 1) contraction of a density feature, 2) coalescence of two density groups, and 3) splitting of one density group into two. In all of these examples, the MEF-estimated flows and pressure fields match well with the "ground-truth" values, as can be seen 


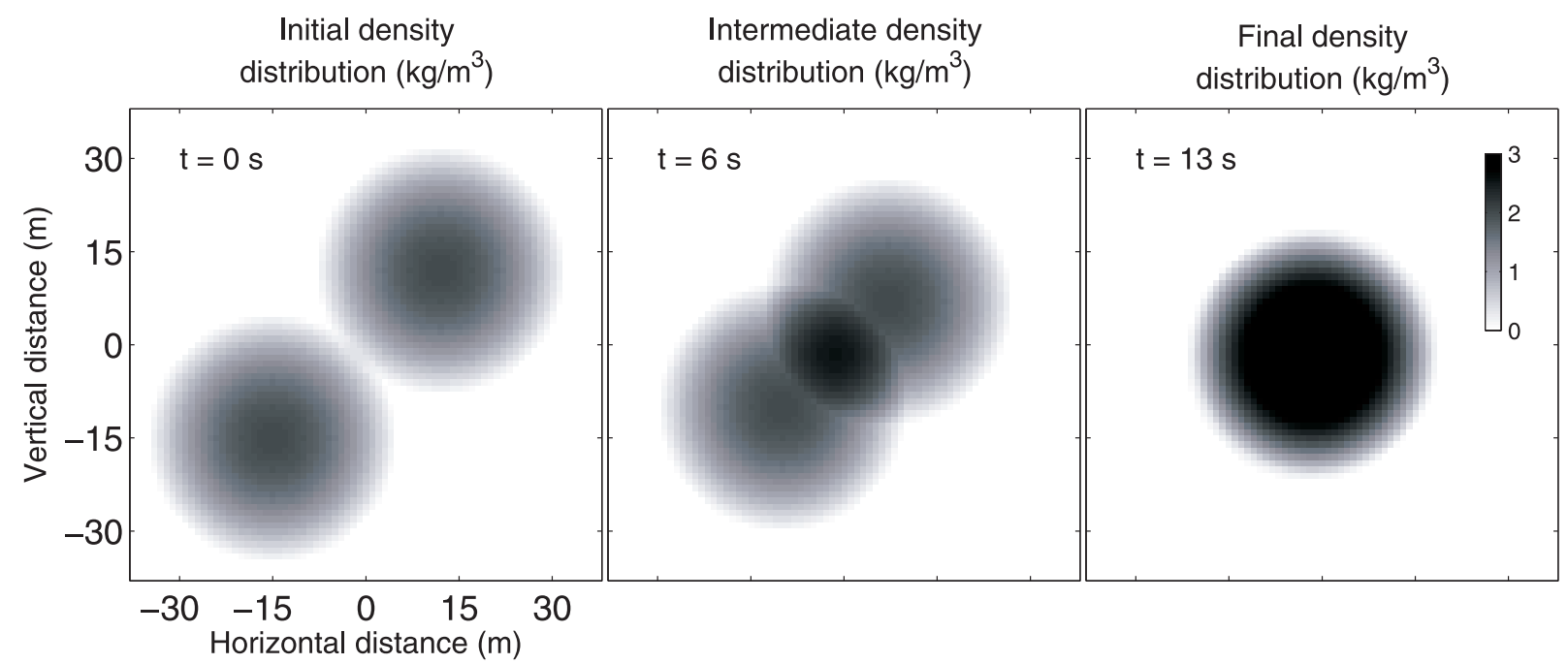

(a)

(b)

(c)

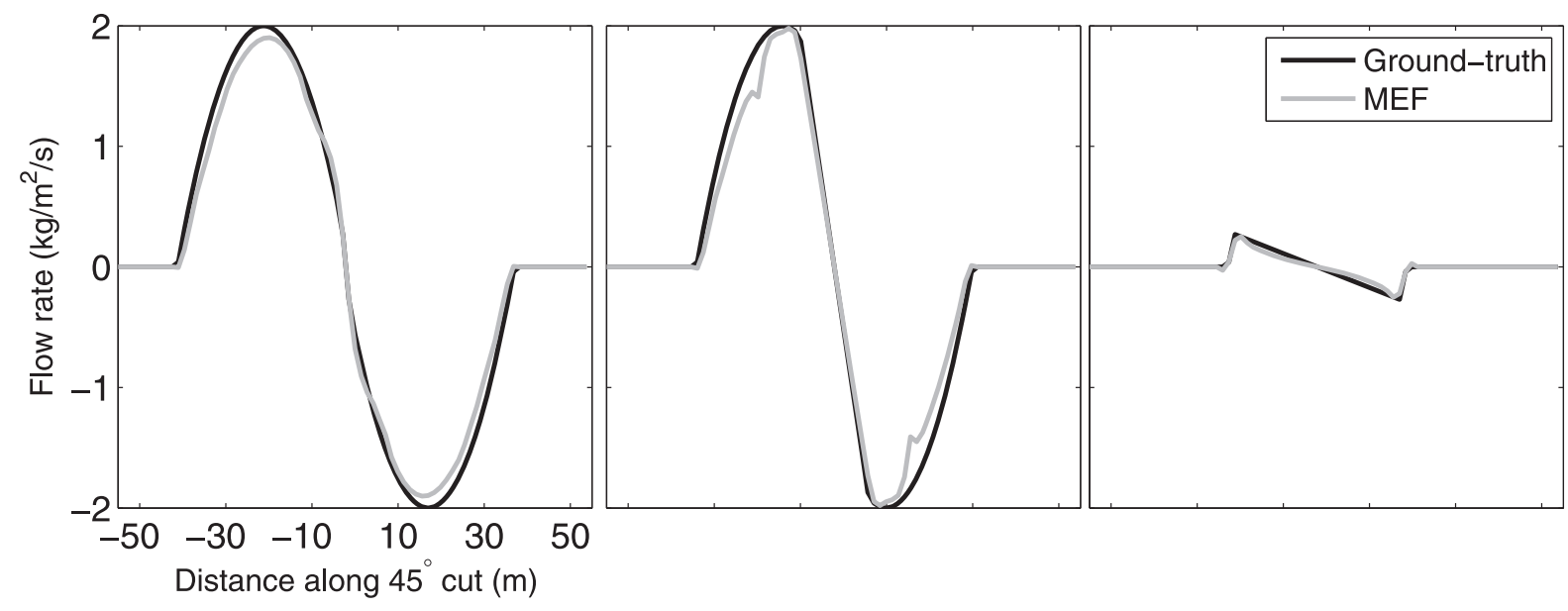

(d)

(e)

(f)

Fig. 2. Example of two density groups coalescing into one. The density distributions during the (a) initial, (b) intermediate, and (c) final stages of coalescence, respectively. (d), (e), (f) Comparison between ground-truth and MEF-computed mass flow rates along a 45 degree cut in (a), (b), and (c), respectively. The maximum error in the MEF-estimated flow is $\sim 10$ percent.

from Figs. 1, 2, and 3. The places where the MEF-estimated mass flow vectors differ the most from the "ground-truth" flows are areas of low density and low-density gradient. This is because MEF, similarly to the traditional Optical Flow method [23], relies on spatial gradients and temporal changes of density to provide information about the underlying motion. In a special case, if the observed images describe a constant flow along iso-density lines, the velocity fields are indeterminate.

In this paper, we use two-dimensional density images and two-dimensional flow fields to illustrate the utility of the force-estimation and MEF techniques. The same techniques can be applied to three-dimensional density images in biomedical imaging systems such as Magnetic Resonance Imaging (MRI) [43] and CT.

\subsubsection{Illustrative Example 1: Contraction of a Density Feature}

Here we consider a circular density feature with a radius, $r$, of $20 \mathrm{~m}$ at $\mathrm{t}=0 \mathrm{~s}$ (Fig. 1a), which contracts uniformly so that its radius at $t=1 \mathrm{~s}$ is $19 \mathrm{~m}$ (Fig. $1 \mathrm{~b}$ ) and at $\mathrm{t}=2 \mathrm{~s}$ is $18 \mathrm{~m}$
(Fig. 1c). The "ground truth" flow fields that result in the changes in density distribution observed in Figs. 1a, 1b, and 1c can be readily computed using pairs of density images, the continuity constraint (1), and the geometrical constraints for this problem

$$
\begin{aligned}
& u=-k x, \\
& v=-k y,
\end{aligned}
$$

where $k=1 / r$. The ground-truth flow at each time step is then computed as the product of the known constant velocity field and the known density distribution. Using the ground truth flows at $t=0 \mathrm{~s}$ and $t=1 \mathrm{~s}$, we then compute the driving pressure field at $t=0 \mathrm{~s}$ (Fig. 1d) using (11) and (12).

We now apply the MEF and force estimation techniques developed in Sections 3.1 and 3.2, to the density image sequence in Figs. 1a, 1b, and 1c. Our MEF-computed flows and pressures are compared to the "ground truth" values in Figs. 1e and 1f, respectively. The maximum error in flow 


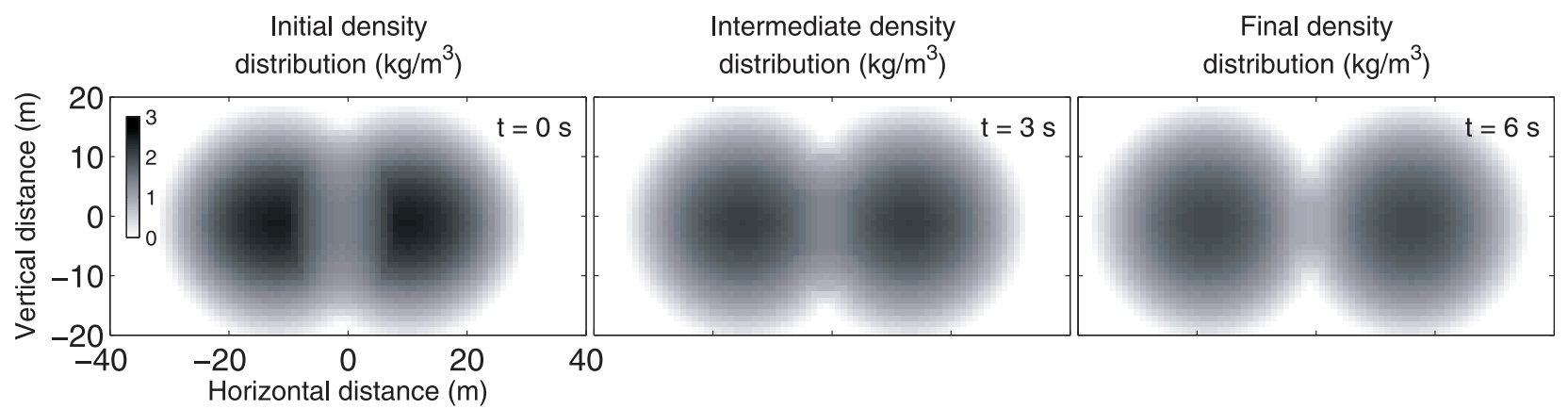

(a)

(b)

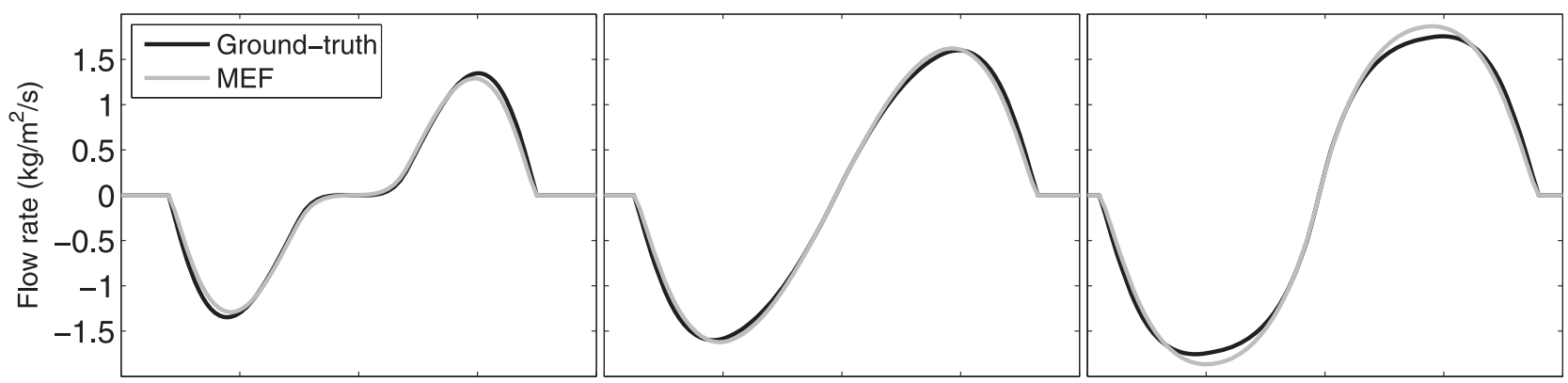

(e)

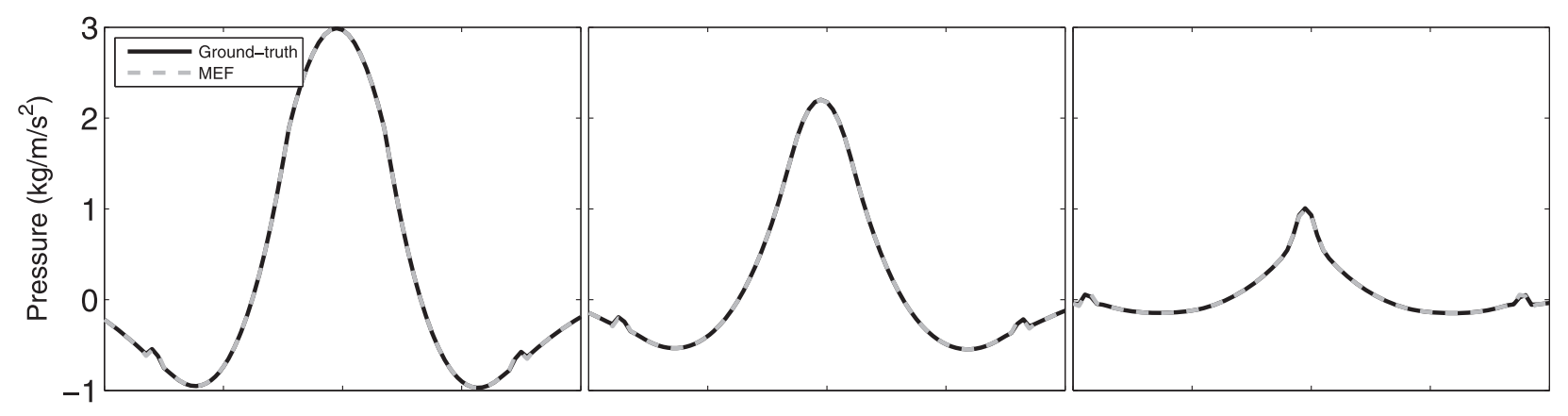

(g)

(h)

(i)

Fig. 3. The density distributions during the (a) initial, (b) intermediate, and (c) final stages of splitting, respectively. (d), (e), (f) Comparison between ground-truth and MEF-computed mass flow rates along a horizontal cut, $y=0$, in (a), (b), and (c), respectively. (g), (h), (i) Comparison between ground truth and MEF-computed pressure along a horizontal cut, $y=0$, in (a), (b), and (c), respectively. The MEF-estimated pressure lies almost exactly on top of the ground-truth pressures. The maximum error in the MEF-estimated flow is less than 5 percent, while the maximum error in our estimated pressure is less than 1 percent.

estimates is less than 5 percent, while the maximum error in the pressure estimate is $\approx 10$ percent.

The type of compressible motion we have chosen in Fig. 1 is commonly encountered in medical imaging, where, for example, CT image sequences describe contraction and expansion of the heart [48] and lungs [21], both of which are elastic deformable objects.

\subsubsection{Illustrative Example 2: Coalescence of Two Density Groups}

Here we consider a sequence of density images that describes a coalescence episode, where two density groups (Fig. 2a) translate toward each other at a constant speed until they merge. The total density at each step and pixel is the algebraic sum of the densities of the two density groups. As seen from Fig. 2a, the two groups are initially $(\mathrm{t}=0 \mathrm{~s})$ separated such that their centers of mass are, respectively, at $(15 \mathrm{~m}, 15 \mathrm{~m})$ and $(-15 \mathrm{~m},-15 \mathrm{~m})$. At $\mathrm{t}=6 \mathrm{~s}$, their centers have moved to $(7.5 \mathrm{~m}, 7.5 \mathrm{~m})$ and $(-7.5 \mathrm{~m},-7.5 \mathrm{~m})$ (Fig. 2b), and finally, at $\mathrm{t}=13 \mathrm{~s}$, they have merged (Fig. 2c). The entire sequence consists of 15 frames, each separated by $\Delta t=1 \mathrm{~s}$. Since the two density groups translate toward each other at a constant speed, there is no external force or pressure that acts on the groups.

The ground truth flow at each time step is computed as the product of the known constant velocity field and the known density distribution. The MEF flow field is computed by using (8) and (9), and corresponding pairs of density distributions $(\rho(t=0), \rho(t=1)),(\rho(t=6), \rho(t=7))$, and $(\rho(t=13), \rho(t=14))$. In Fig. 2, we compare MEF and ground-truth flows during the initial (Figs. 2a and 2d), intermediate (Figs. $2 \mathrm{~b}$ and 2e), and final (Figs. 2c and 2f) stages of the coalescence episode. The maximum error in the MEF-estimated flow is $\approx 10$ percent.

The example in Fig. 2 illustrates the application of MEF to estimate both incompressible translation (Figs. 2a and 2d) and compressible coalescence (Figs. 2c and 2f). These motion types are commonly encountered in quantifying cloud field 


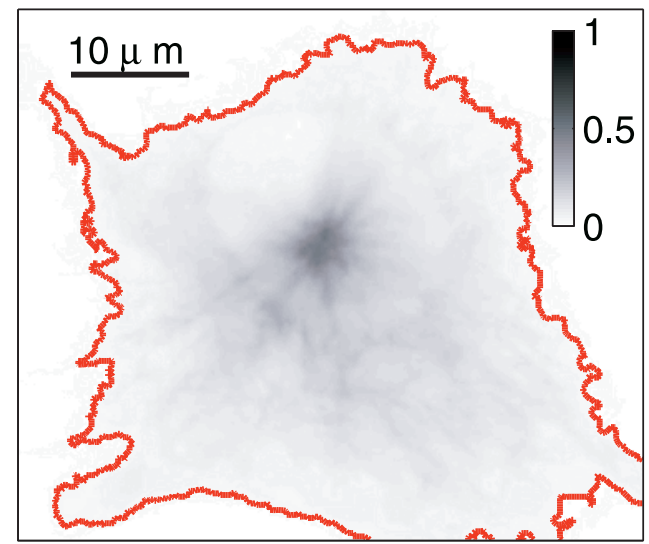

(a)
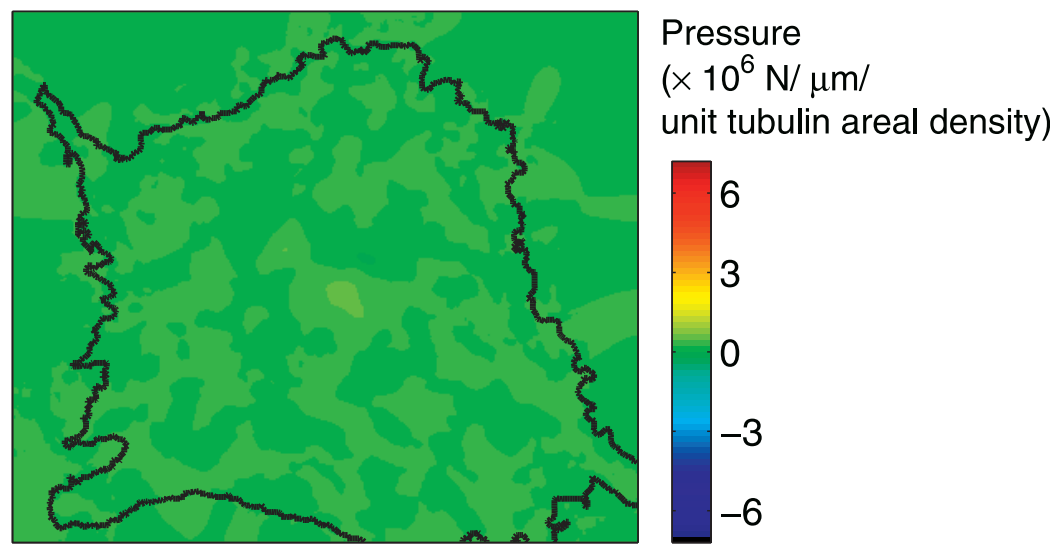

(b)

Fig. 4. (a) Xenopus laevis cell before undergoing mitosis. The colorscale corresponds to the relative areal density of a flourescent marker, GFP alpha-tubulin, which attaches itself to structures called microtubules. The density is normalized so that the maximum number of tubulin per square $\mu \mathrm{m}$ is 1 in Fig. $5 \mathrm{c}$. The red contour represents the cell boundary (cytoplasm). (b) Pressure distribution inside a Xenopus laevis cell prior to mitosis. The pressures are one order of magnitude smaller compared to those in Fig. $5 \mathrm{f}$.

kinematics using satellite images [8] and, as we shall see in Section 4.3, in imaging large fish shoals [32] using OAWRS.

\subsubsection{Illustrative Example 3: Splitting of Density Groups}

The final example we consider for evaluating the MEF and force-estimation techniques is a density image sequence describing the splitting of one density group into two. In this example, a single dense group (Fig. 3a) splits into two (Figs. $3 \mathrm{~b}$ and $3 \mathrm{c}$ ) over a time frame of $6 \mathrm{~s}$. The "groundtruth" flows and pressures (black solid lines in Figs. 3d, 3e, $3 \mathrm{f}, 3 \mathrm{~g}, 3 \mathrm{~h}$, and $3 \mathrm{i}$ ) are computed at each time step using the procedure described in Appendix D. We also apply the MEF and force-estimation techniques to the density image sequence and estimate the flows and pressures (gray lines in Figs. 3d, 3e, 3f, 3g, 3h, and 3i).

The maximum error in the MEF-estimated flow is less than 5 percent (Figs. 3d, 3e, and 3f), while the maximum error in our estimated pressure is less than 1 percent.

Image sequences describing splitting of density groups, such as the example we have chosen in Fig. 3, are encountered in imaging systems that capture cell division, such as Flourescent Speckle Microscopy [11]. We will show an application of the MEF and force-estimation techniques in Section 4.2, where we quantify the mass flows and pressure distribution inside a cell undergoing mitotic cell division.

\subsection{Quantifying Velocity and Force Fields Driving Cell Division}

Here, we quantify the dynamics of cell division using the MEF and force-estimation techniques developed in Sections 3.1 and 3.2. Currently, it is hypothesized [25], [26] that intracellular forces driving cell division are generated by long, fiber-like structures called microtubules. It is also postulated that the microtubules pull apart newly formed chromosome pairs by generating a combination of repulsive forces at the center and attractive forces at the poles of the cell [26], [30]. While several molecular mechanisms have been proposed for force generation [30], it has been difficult to quantify these forces and their distribution within the cell, prompting the need for "a combination of bio-physical force measuring methods and molecular biological mutagenesis methods" [30].
By applying the MEF and force-estimation techniques to an image sequence describing mitosis (the process by which a cell replicates itself by splitting in two), we quantify intracellular forces driving cell division. We use an image sequence describing mitosis in a Xenopus laevis [40] cell (Fig. 4a). The cell has been injected with a fixed amount of a flourescent marker called GFP alpha-tubulin [11]. The colorscale in Fig. 4a is proportional to the areal number density of GFP alpha-tubulin [11]. Before the cell splits, the velocity field inside the cell is random and has a small magnitude (on the order of $0.1 \mu \mathrm{m} / \mathrm{s}$ ) compared to the velocity field during mitosis (Fig. 5).

Figs. 5a, 5b, and 5c describe "Anaphase" [19], one of the four stages in mitosis, where newly formed chromosome pairs [19] within the cell are pulled apart, resulting in cell division. Using the density image sequence (Figs. 5a, 5b, and $5 c)$, we compute the velocity field that describes the effective dynamics of the fluorescent tubulin within the cell (Fig. 5d). The velocity vectors indicate a tubulin flux toward opposite ends of the cell at rates of $2 \mu \mathrm{m} / \mathrm{s}$, which is consistent with previous velocity estimates [30]. Using the velocity field, we then compute the net force density (i.e., the right-hand side of (11) and (12)) driving cell division (Fig. 5e). The maximum areal density of tubulin in our density images is $1.5 \times 10^{-14} \mathrm{~kg} / \mu \mathrm{m}^{2}$, and is computed using an intertubulin spacing of $4 \mathrm{~nm}$ [30] within a microtubule, a molecular mass of $55 \mathrm{kDa}\left(55 \times 1.66 \times 10^{-24} \mathrm{~kg}\right)$ for tubulin and a typical cell thickness of $10 \mu \mathrm{m}$ [39]. We find that the magnitudes of our net force density vectors are comparable with experimentally measured values of force exerted by microtubules on glass microbeads (0.2 pico $\mathrm{N}$ ) [14].

In order to compute our intracellular forces, we have made a continuum assumption that is suitable for fluid motion. In the case of cell division, such a fluid assumption may still be applicable, given the semiflexible nature [28] of microtubules that are suspended and moving in a cytoplasmic fluid. It should also be noted that the net force density may include components arising from the elasticity of microtubules, which can be estimated only by including additional constraints in our force model. We find the difference in total tubulin density between Figs. $5 \mathrm{a}$ and $5 \mathrm{c}$ to be less than 10 percent, suggesting that the approximation we made in 


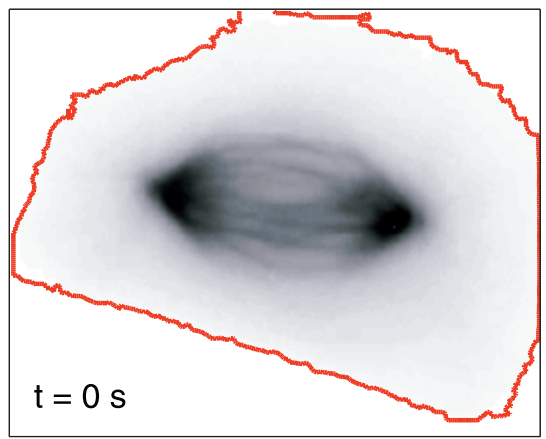

(a)

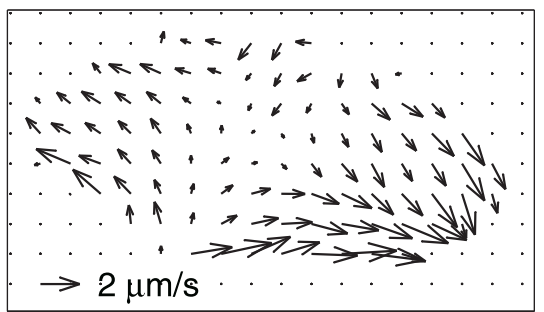

(d)

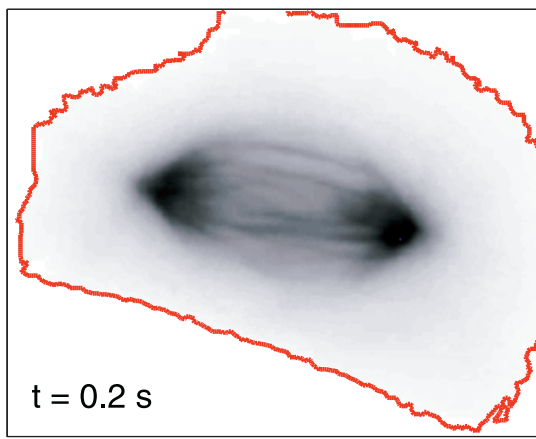

(b)

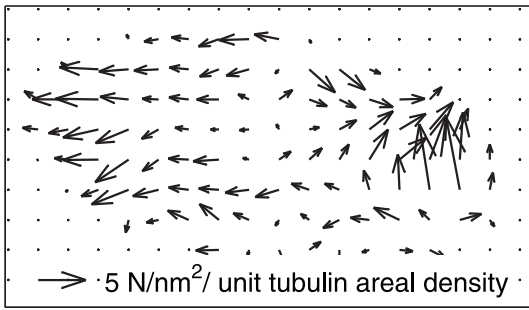

(e)

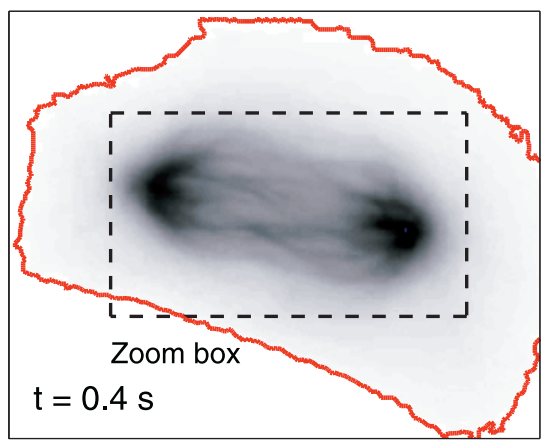

(c)

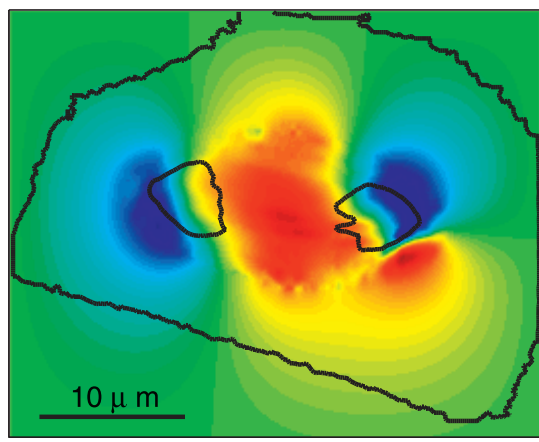

(f)

Fig. 5. (a), (b), (c) Sequence of frames showing mitosis in a Xenopus laevis cell. Same colorscale as that in Fig. 4a. Cell boundary is marked by red contours. The black box in (c) is the area zoomed in (d), (e). Note long fiber-like structures called microtubules. (d) Velocity field derived from density image pairs (a), (b). The vectors are shown every 10 pixels. (e) Net force density computed using velocity and density fields in (11) and (12). (f) Pressure field that gives rise to the force field in (e). Same colorscale as in Fig. 4b. Formation of two low apparent pressure regions at the opposite ends of the cell and a high apparent pressure region at the center of the cell is shown. Two regions of high microtubule density and the cell boundary are shown as black contours.

neglecting source and sink terms in our fomulation is a good one for this problem. Such source or sink terms may arise due to polymerization or depolymerization of tubulin molecules, and can be easily included in (1).

Under our assumptions of fluid flow in a cell, the net force is the result of the effective pressure field shown in Fig. 5f. We find that cell division is driven by the formation of two regions of low apparent pressure at opposite sides of the cell, and a region of high apparent pressure at the center. This is in contrast to the random pressure field inside the cell before mitosis (Fig. 4b), which has a much smaller magnitude. These effective pressures are different from the hydrodynamic pressures related to the flow of the cytoplasmic fluid. The visualization of pressure shown in Fig. 5f quantifies the repulsive force field at the center as well as the attractive force fields at opposite poles of the cell. Such force fields have been previously postulated to drive cell division [26], [30].

\subsection{Application to Fish Population Density Images}

We now apply the MEF and force-estimation techniques developed in Sections 3.1 and 3.2 on fish population density images obtained using an Ocean Acoustic Waveguide Remote Sensing system, to quantify flow rates and pressure fields driving the dynamics of large fish shoals. Using the MEF-computed flow fields, we quantify the behavior of large fish shoals including 1) translation and coalescence of fish groups, and 2) mass exchange between different parts of a large shoal via hourglass patterns.
The OAWRS system has been recently developed [32] to detect, image, and continuously monitor large fish shoals over continental shelf-scale areas. It consists of a source that transmits low-frequency sound in the audible frequency range, which is trapped between the ocean-air and oceanseabed boundaries as it propagates over long distances and scatters off fish shoals and other submerged targets. These scattered returns are collected by a towed receiver and charted in range and bearing, resulting in an instantaneous snapshot of the ocean over hundreds of square kilometers. The intensity of the scattered returns from fish shoals is proportional to the fish population density [3], [27], so that, by repeating transmissions at regular intervals, a population density image sequence is generated. A detailed technical description of the OAWRS system can be found in [20], [27], [32], [33].

An example of the type of population density image obtained using OAWRS is shown in Fig. 6, which shows a large shoal of fish centered roughly $12 \mathrm{~km}$ south and $5 \mathrm{~km}$ east of the source. This image was obtained on 14 May 2003, off the coast of New Jersey during the OAWRS 2003 experiment [32]. The shoal was observed for an entire day using OAWRS, which provided snapshots of population density every $50 \mathrm{~s}$. We will apply MEF to the sequence of fish population density images in an area defined by the box in Fig. 6 .

To compute force fields using (11) and (12), we assume that individual fish behave like fluid particles so that the entire fish shoal (Fig. 6) behaves like an anisotropic, compressible fluid. This assumption is consistent with OAWRS observations of spatial and temporal variation of 


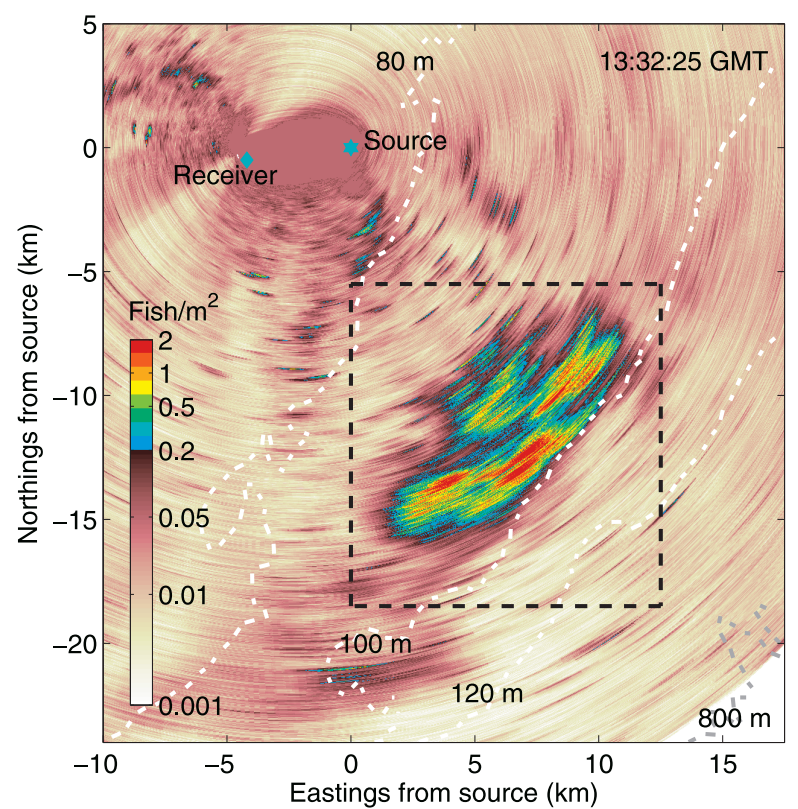

Fig. 6. Large shoal of fish imaged off the New Jersey coast on 14 May 2003 using OAWRS. The colorscale represents the areal density of the fish. The image resolution is $30 \mathrm{~m} / \mathrm{pixel}$. The bathymetric contours are shown using white dashed lines. The black dashed box is the area over which MEF and force-estimation techniques are applied to study the dynamics of the large shoal and is the area shown in Figs. 7 and 10.

population density, which showed that fish could converge or diverge, making their motion highly compressible. Similar observations of fish schools behaving like an "animate fluid" [10] have been reported for small schools of a few meters in extent.

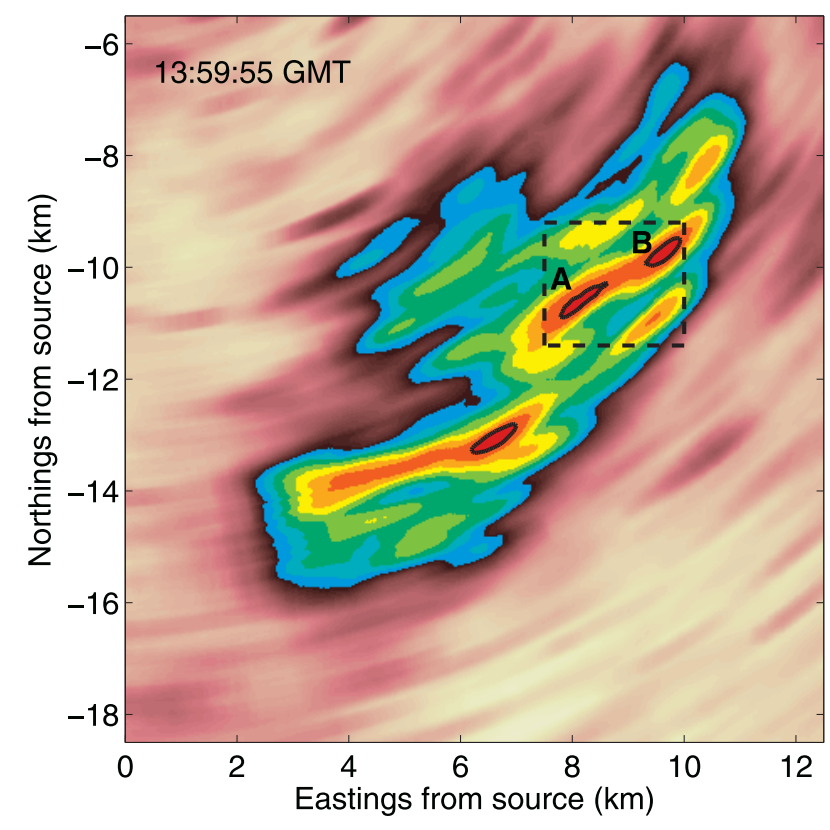

Fig. 7. Fish population density image showing schools A and B before merger. Same colorscale as in Fig. 6. The original OAWRS density image has been smoothed such that the areal density at any point in the image shown above, is the unweighted mean of the areal densities over a $120 \mathrm{~m} \times 120 \mathrm{~m}$ square area centered at that point. The dashed box represents the zoom area over which velocity vectors are shown in Fig. 8. Black lines are 1.5 fish $/ \mathrm{m}^{2}$ population density contours.

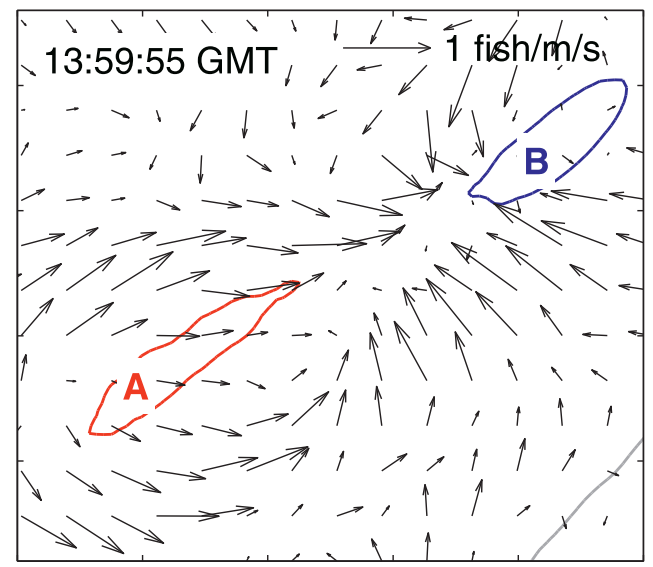

(a)

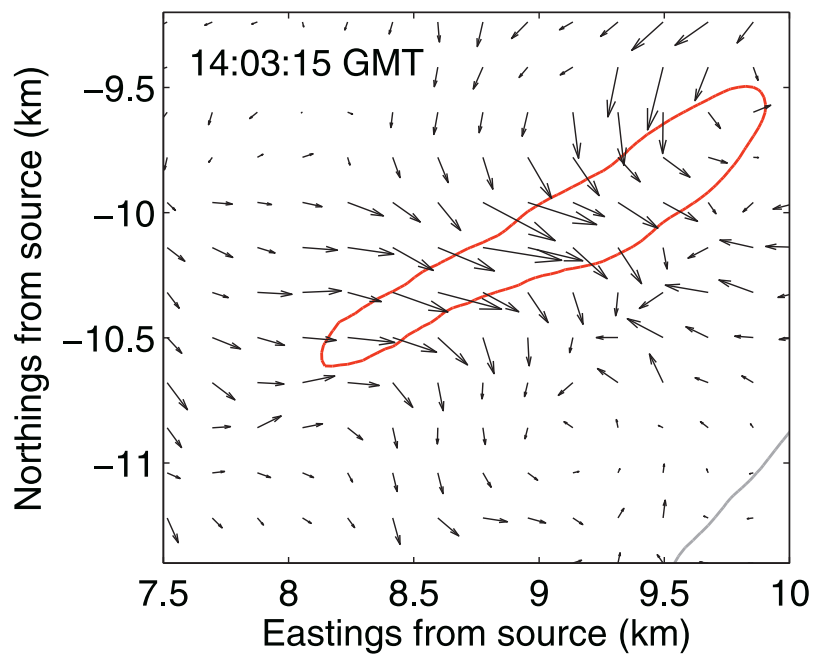

(b)

Fig. 8. (a) Flow vectors describing the merger of groups A (marked in red) and $B$ (marked in blue). Blue and red lines represent the $1.5 \mathrm{fish} / \mathrm{m}^{2}$ population density contours. The gray line represents the $0.2 \mathrm{fish} / \mathrm{m}^{2}$ population density contour. (b) Flow field after the merger of $A$ and $B$. The red line represents the $1.5 \mathrm{fish} / \mathrm{m}^{2}$ population density contour. The groups merge within a span of 3 minutes. The mass flow vectors are shown every 10 pixels or $300 \mathrm{~m}$.

Under our continuum assumptions, the net force can be thought of as the result of a pressure field, with regions of low pressure acting as centers of attraction and regions of high pressure acting as centers of repulsion. These pressures are different from the hydrodynamic pressures related to the flow of water in the ocean. They are effective biological stresses that drive fish shoaling behavior.

\subsection{Translation and Coalescence of Fish Groups}

Here, we use the MEF and force-estimation techniques to quantify the rates at which fish groups within a large shoal translate and coalesce. We find that the rate of translation is consistent with the swimming speeds of individual fish. We also find that coalescence of fish groups can occur due to formation of "attraction zones" or regions of low pressure. These phenomena are quantified by tracking the motion of two high population density regions, A and B, shown in Fig. 7 . The MEF-estimated velocity vectors, shown in Fig. 8, describe the translation and coalescence of $\mathrm{A}$ and $\mathrm{B}$, occurring at rates of roughly $0.5-1 \mathrm{~m} / \mathrm{s}$. The merger of $A$ and $B$ can also be 


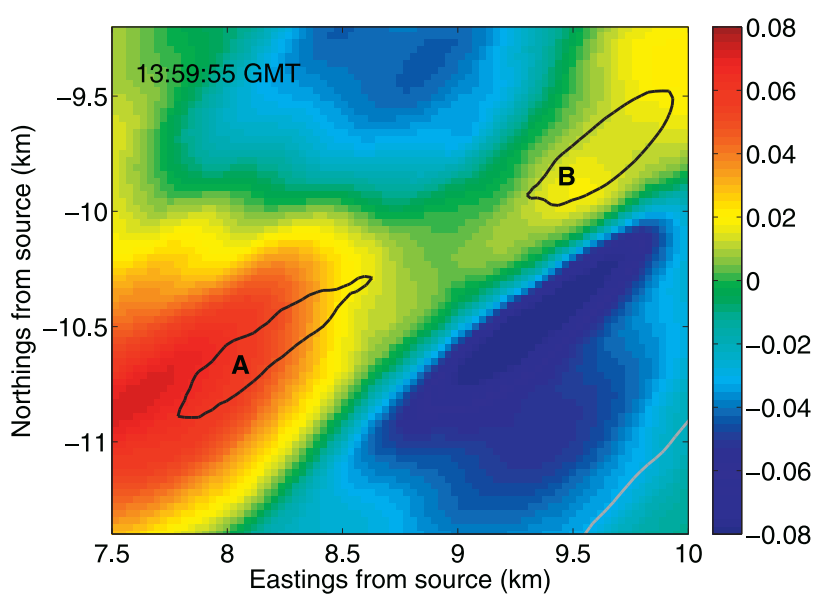

Fig. 9. Pressure $\left(\mathrm{N} / \mathrm{m}^{2}\right.$ per unit fish mass) distribution within large fish shoal showing formation of a low-pressure region that attracts schools $A$ and B. Black lines represent the $1.5 \mathrm{fish} / \mathrm{m}^{2}$ population density contours. The gray line represents the $0.2 \mathrm{fish} / \mathrm{m}^{2}$ population density contour. Same zoom area as Fig. 8.

thought of as the result of a low pressure, "attraction zone" formed between the schools, as shown in Fig. 9.

The mean velocity of groups $A$ and $B$ can also be estimated by tracking their centers of mass (COM) defined by

$$
\begin{aligned}
& \bar{X}_{A}=\frac{\sum_{i \in A} \rho_{i} x_{i}}{\sum_{i \in A} \rho_{i}}, \quad \bar{Y}_{A}=\frac{\sum_{i \in A} \rho_{i} y_{i}}{\sum_{i \in A} \rho_{i}}, \\
& \bar{X}_{B}=\frac{\sum_{i \in B} \rho_{i} x_{i}}{\sum_{i \in B} \rho_{i}},
\end{aligned}
$$

where $i$ represents the pixel number. We find that group A moves toward group $B$ at roughly $1 \mathrm{~m} / \mathrm{s}$, which is

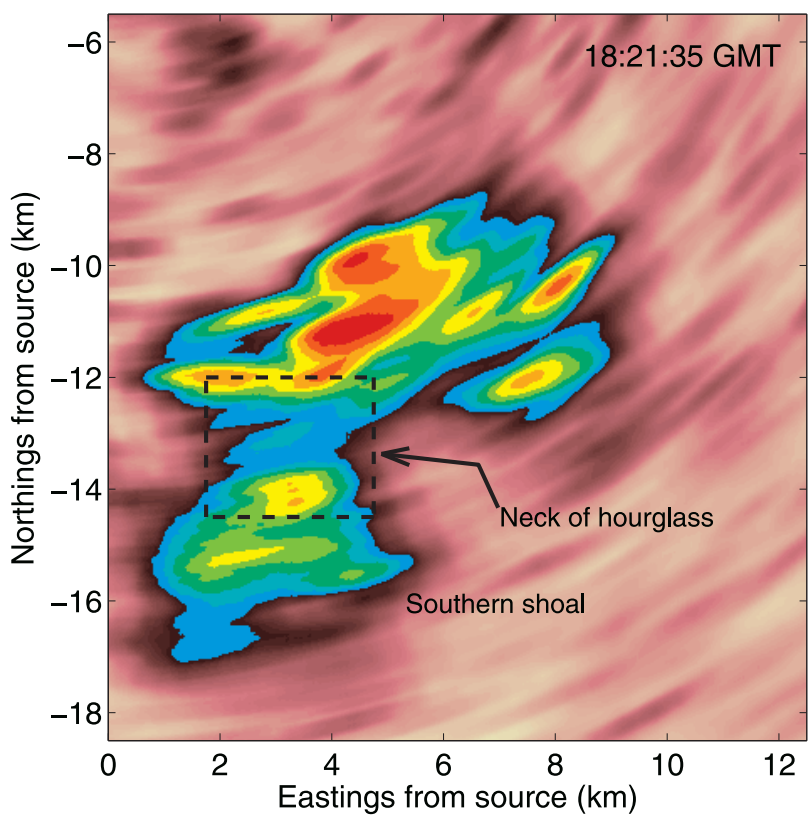

Fig. 10. Fish density distribution showing hourglass type formation. Same colorscale as in Fig. 6 . The southern shoal gets depopulated and there is mass flow across the neck of the hourglass shown. The black box is the area zoomed in Figs. 11 and 12. The areal density has been smoothed using the same algorithm as that employed in Fig. 7.

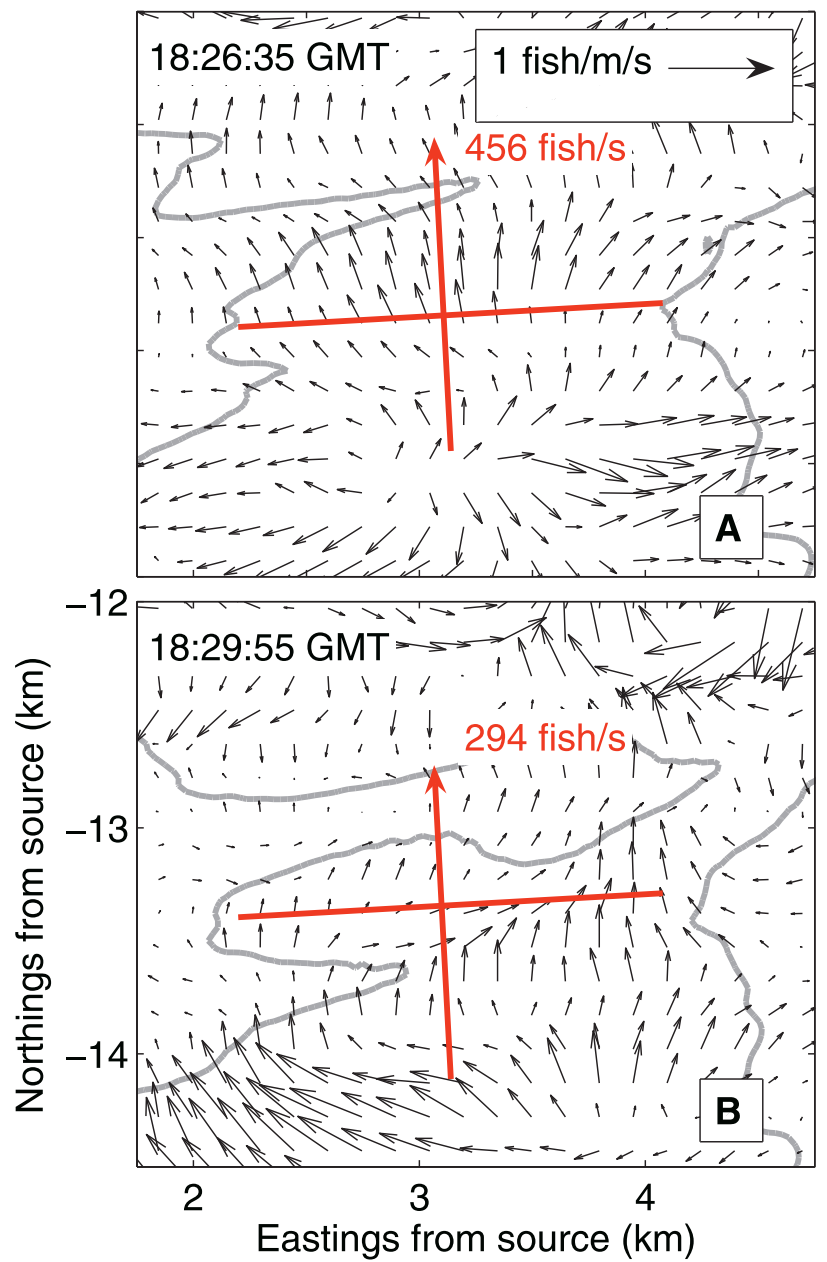

Fig. 11. Mass flow distribution frames showing depopulation of the southern wing over a span of 3 minutes. The area shown is zoomed around the neck of the hourglass shown in Fig. 10. The flow rate of fish normal (red arrow) to the neck (red solid line) is found to be $\sim 300-450$ fish/s. The flow vectors are shown every 5 pixels. The gray lines are $0.2 \mathrm{fish} / \mathrm{m}^{2}$ density contours.

consistent with the velocities obtained using MEF (Fig. 8). These values are also consistent with the typical speeds at which individual fish swim [16], [24], [36].

\subsection{Mass Exchange between Different Parts of a Shoal}

We now quantify fish flow rates between different parts of the large shoal shown in Fig. 6. In particular, we quantify the rate of mass transfer between two wings of an hourglass pattern formed by the fish shoal, as shown in Fig. 10. We find that there is a steady depopulation of the southern wing and the fish "flow" into the northern wing, as can be seen from the sequence of images in Fig. 11. There is a steady flow of $\sim 300-450 \mathrm{fish} / \mathrm{s}$ across the neck of the hourglass connecting the two wings of the shoal. The depopulation episode can also be explained by the formation of a high-pressure region near the neck of the hourglass (Fig. 12).

Hourglass patterns have been observed in smaller fish groups spanning spatial scales on the order of a square $\mathrm{km}$ [42]. Mass transfers of the kind described above have been known to occur and have been shown in these small groupings. Flow from one part of the shoal to the other via 


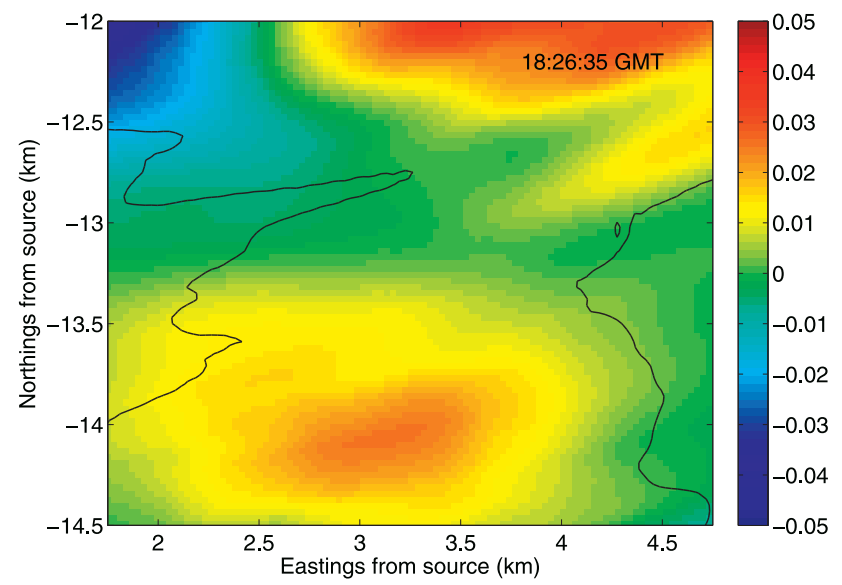

Fig. 12. Pressure $\left(\mathrm{N} / \mathrm{m}^{2}\right.$ per unit fish mass) distribution within a large fish shoal showing formation of a high-pressure region near the "neck" of an hourglass pattern, forcing fish mass flow from one wing to the other. The black lines are $0.2 \mathrm{fish} / \mathrm{m}^{2}$ density contours.

the "neck" usually signifies predatory pressure on one of the wings [42]. The depopulation described by the MEF calculation could very well be in response to such a pressure acting on the southern wing of the large shoal described by the OAWRS density images.

\section{Prediction Using Forces: Application to SYNTHETIC IMAGES}

Here we apply the prediction procedure shown in Section 3.3 to density images in Fig. 1, where a circular feature undergoes uniform contraction.

In Fig. 1, we considered density images for $t=0,1$, and $2 \mathrm{~s}$, and computed the flow field and pressure field driving contraction. We now continue this contraction, and predict the density distribution at times $t=3-7 \mathrm{~s}$ (Fig. 13). Comparison of our predicted densities with actual values (Fig. 13) shows a good match (errors $<10$ percent) until $t=7 \mathrm{~s}$, after which the cumulative effect of errors becomes large and causes significant (errors $>10$ percent) difference between predicted and actual densities.

In general, we expect our prediction scheme to work well within some time interval for cases where the pressures and forces driving the flow remain more or less constant for the time interval. This is indeed the case in many natural flows which follow environmental pressure gradients, such as the movement of clouds in the atmosphere driven by the formation of low and high-pressure regions.

\section{Conclusions}

We have presented methods for 1) estimating forces that drive motion observed in density image sequences and 2) predicting flow and density evolution. To do this, we developed a Minimum Energy Flow method for estimating velocity fields in both compressible and incompressible flows. The MEF and force-estimation techniques have been demonstrated with synthetic and experimentally obtained images. Using a density image sequence describing cell mitosis, we showed that cell division is driven by gradients in apparent pressure in the cell. Using density image

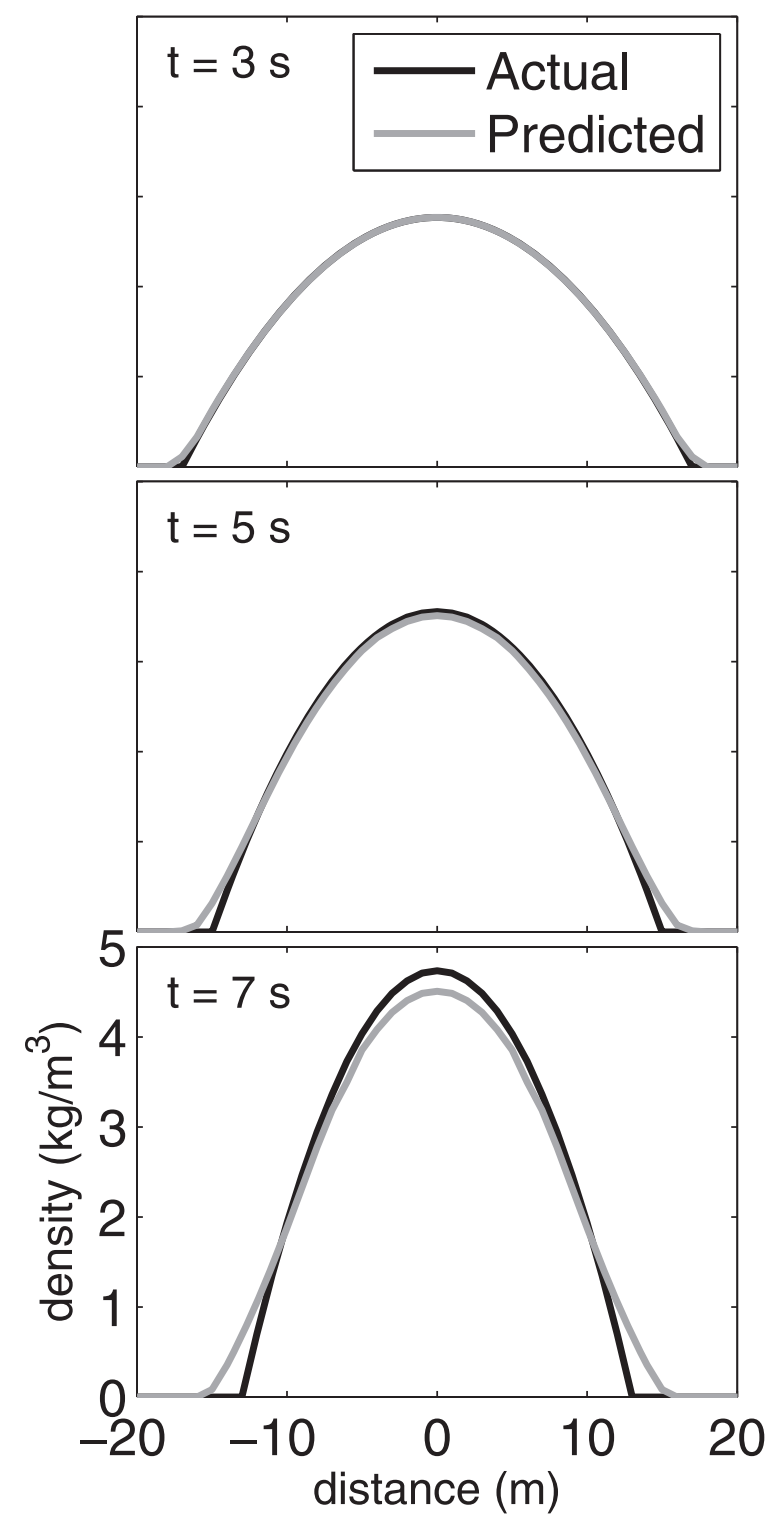

Fig. 13. Comparison of actual and predicted densities for different times. The same example as that in Fig. 1 is used. The curves are cuts through $y=0$ in the actual and predicted density images. The prediction scheme works well within some time interval when the forces remain more or less constant. After some time, the cumulative effect of errors becomes large and causes a significant (errors $>10$ percent) difference between predicted and actual densities.

sequences of fish shoals, we also quantified 1) coalescence of fish groups over tens of kilometers, 2) fish mass flow between different parts of a large shoal, and 3) the stresses acting on large fish shoals.

The MEF and force estimation techniques can be generally applied to any density image sequence where pixel values can be modeled as proportional to the density of a compressible fluid. In addition to the examples presented here, such density image sequences are frequently encountered in biomedical imaging and satellite imaging for meteorology and oceanography. MRI, for example, provides tomography image sequences of blood flow in arteries, which could be monitored using our MEF and force-estimation techniques. Satellite images of density distribution of water vapor (clouds), for example, can be 


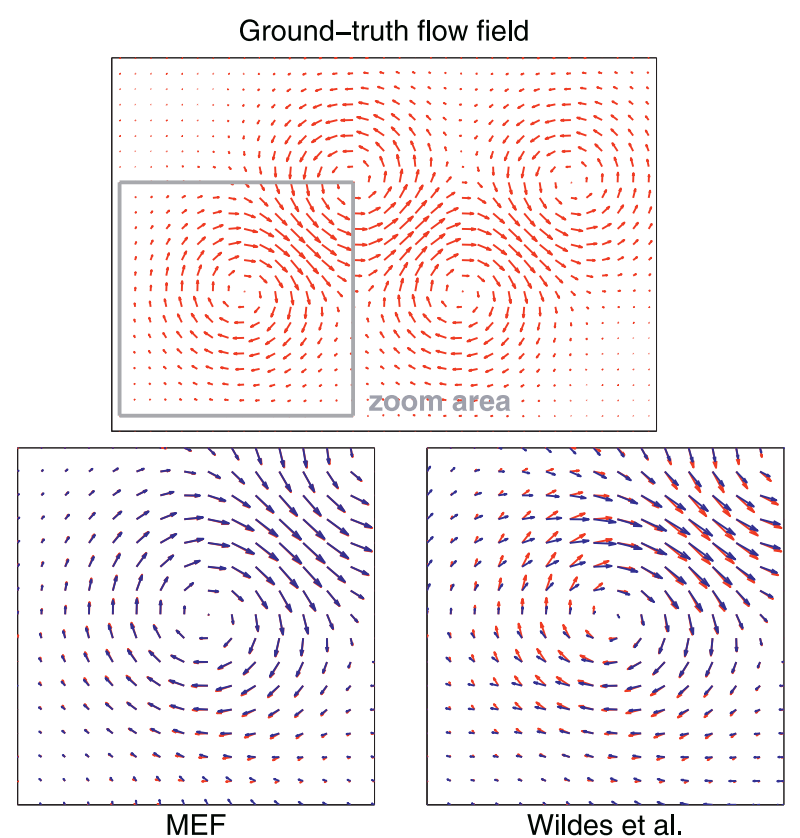

Fig. 14. Comparison of MEF and the method proposed in [56]. (Top) Ground-truth flow field-an idealization of a von-Kármán vortex street. (Bottom left) Comparison between MEF-estimated (blue) and groundtruth mass flows in the zoom region shown in (Top). The vectors lie almost on top of each other and the maximum error is $\sim 10$ percent. (Bottom right) Comparison between flow vectors estimated using the method proposed by Wildes et al. (blue arrows) and the ground-truth vectors (red arrows). There is significant error ( $\sim 30-40$ percent) in the estimated vectors.

used to compute flow and force fields in the atmosphere that drive meteorological processes. Other applications are in studies of collective behavior, where the MEF and forceestimation tools can be used to verify theoretical models that predict average velocities and forces acting in large animal groups.

\section{APPENDIX A}

\section{Comparison of MEF with the Method Proposed BY WILDES ET AL.}

Here, we compare the performance of MEF and the method proposed by Wildes et al. [56], in recovering motion involving large changes in velocity over space. As mentioned in Section 2, we expect the latter to "smooth out" large variations and the former to preserve these variations. For flows that involve small variations in velocity over space, both of these methods are expected to perform equally well.

In this section, we quantify the ability of both methods to recover an idealization of a Kármán vortex street [5], which is a good example of a flow with large spatial gradients in velocity, as illustrated in Fig. 14. Such a repeating pattern of swirling vortices is caused by the unsteady separation of flow of a fluid over bluff bodies [5]. Accurately quantifying vortices is important in many fields such as medical imaging of blood flow using MRI, where the presence of vortices, for example, indicates blockages of arteries [44]. Here, we have idealized each vortex in Fig. 14 as a "LambOseen vortex" [45], which models a line vortex that decays due to viscosity. The tangential velocity of the vortex is given as a function of radius $r$

$$
V_{\theta}(r)=\left(V_{\theta, \max }\right)\left(1+\frac{0.5}{\alpha}\right) \frac{r_{c}}{r}\left[1-\exp \left(-\frac{\alpha r^{2}}{r_{c}^{2}}\right)\right],
$$

where $V_{\theta, \max }$ is the peak tangential velocity, $\alpha$ is a viscositydependent constant, and $r_{c}$ is the core radius of the vortex. In this example, we have chosen $V_{\theta, \max }=1, \alpha=1.26$ [12], and $r_{c}=10$ for each vortex shown in Fig. 14 .

In the example we have chosen, the MEF technique recovers the motion to within 10 percent accuracy except in regions of very low velocity, as can be seen from Fig. 14 . This contrasts with the method proposed by Wildes et al., where errors are high (30-40 percent) even in regions of high velocity (Fig. 14) and shows that the "unsmoothness of flow" criterion chosen in [56] distorts the flow field in order to make it vary more smoothly than in the actual flow.

Corpetti et al. have employed a more complicated "divcurl minimization" technique [9] to preserve vortices in the flow field, rather than the Principle of Least Action used here. They report errors on the order of 10 percent [9] when recovering vortices in fluid flow, as we find here for the simpler MEF approach.

\section{APPENDIX B}

\section{Discretization AND NUMERICAL IMPLEMENTATION OF MEF}

In order to solve (8) and (9) numerically on a discrete grid, we employ a finite difference method to approximate the partial derivatives.

For this purpose, we use the following "computational stencils":

$$
\begin{aligned}
\left(\bar{u}_{x x}\right)_{i, j} & =\frac{\bar{u}_{i, j+1}-2 \bar{u}_{i, j}+\bar{u}_{i, j-1}}{\epsilon^{2}} \\
\left(\bar{u}_{x y}\right)_{i, j} & =\frac{\bar{u}_{i+1, j+1}-\bar{u}_{i-1, j+1}-\bar{u}_{i+1, j-1}+\bar{u}_{i-1, j-1}}{4 \epsilon^{2}}, \\
\left(\bar{v}_{y y}\right)_{i, j} & =\frac{\bar{v}_{i+1, j}-2 \bar{v}_{i, j}+\bar{v}_{i-1, j}}{\epsilon^{2}} \\
\left(\bar{v}_{x y}\right)_{i, j} & =\frac{\bar{v}_{i+1, j+1}-\bar{v}_{i-1, j+1}-\bar{v}_{i+1, j-1}+\bar{v}_{i-1, j-1}}{4 \epsilon^{2}}
\end{aligned}
$$

where the subscripts $i$ and $j$ are row and column indices, respectively, and $\epsilon$ is the grid interval.

Replacing the spatial partial derivatives in (8) and (9) with finite differences and grouping the terms in $\bar{u}_{i, j}$ and $\bar{v}_{i, j}$, we obtain

$$
\begin{gathered}
\left(\frac{\lambda}{\rho_{i, j}}+\frac{2}{\epsilon^{2}}\right) \bar{u}_{i, j}=\left(\rho_{t x}\right)_{i, j}+\frac{\bar{u}_{i, j-1}+\bar{u}_{i, j+1}}{\epsilon^{2}}+\left(\bar{v}_{x y}\right)_{i, j}, \\
\left(\frac{\lambda}{\rho_{i, j}}+\frac{2}{\epsilon^{2}}\right) \bar{v}_{i, j}=\left(\rho_{t y}\right)_{i, j}+\frac{\bar{v}_{i-1, j}+\bar{v}_{i+1, j}}{\epsilon^{2}}+\left(\bar{u}_{x y}\right)_{i, j} .
\end{gathered}
$$

Based on (B-27) and (B-28), we suggest an iterative algorithm: 


$$
\begin{gathered}
\left(\frac{\lambda}{\rho_{i, j}}+\frac{2}{\epsilon^{2}}\right) \bar{u}_{i, j}^{(n+1)}=\left(\rho_{t x}\right)_{i, j}+\frac{\bar{u}_{i, j-1}^{(n)}+\bar{u}_{i, j+1}^{(n)}}{\epsilon^{2}}+\left(\bar{v}_{x y}\right)_{i, j}^{(n)}, \\
\left(\frac{\lambda}{\rho_{i, j}}+\frac{2}{\epsilon^{2}}\right) \bar{v}_{i, j}^{(n+1)}=\left(\rho_{t y}\right)_{i, j}+\frac{\bar{v}_{i-1, j}^{(n)}+\bar{v}_{i+1, j}^{(n)}}{\epsilon^{2}}+\left(\bar{u}_{x y}\right)_{i, j}^{(n)},
\end{gathered}
$$

where the superscripts $(n+1)$ and $(n)$ represent the iteration numbers.

\section{APPENDIX C}

\section{Solving for Pressure and Force Field}

In order to solve (14) and (15), we rewrite them as

$$
\begin{aligned}
& \left(f_{1}\right)_{y y}=g(x, y, t)+\left(f_{2}\right)_{x y}, \\
& \left(f_{2}\right)_{x x}=h(x, y, t)+\left(f_{1}\right)_{x y} .
\end{aligned}
$$

We now write the spatial derivatives of $f_{1}$ and $f_{2}$ at each pixel $(i, j)$ using finite differences as

$$
\begin{gathered}
\left(\left(f_{1}\right)_{y y}\right)_{i, j}=\frac{\left(f_{1}\right)_{i+1, j}+\left(f_{1}\right)_{i-1, j}-\left(2 f_{1}\right)_{i, j}}{\epsilon^{2}}, \quad(\mathrm{C}-33) \\
\left(\left(f_{1}\right)_{x y}\right)_{i, j}=\frac{\left(f_{1}\right)_{i+1, j+1}+\left(f_{1}\right)_{i-1, j-1}-\left(f_{1}\right)_{i+1, j-1}-\left(f_{1}\right)_{i-1, j+1}}{4 \epsilon^{2}},
\end{gathered}
$$

$$
\left(\left(f_{2}\right)_{x x}\right)_{i, j}=\frac{\left(f_{2}\right)_{i, j+1}+\left(f_{2}\right)_{i, j-1}-\left(2 f_{2}\right)_{i, j}}{\epsilon^{2}}
$$

$\left(\left(f_{2}\right)_{x y}\right)_{i, j}=\frac{\left(f_{2}\right)_{i+1, j+1}+\left(f_{2}\right)_{i-1, j-1}-\left(f_{2}\right)_{i+1, j-1}-\left(f_{2}\right)_{i-1, j+1}}{4 \epsilon^{2}}$.

Based on the above finite difference scheme, we suggest the following iterative procedure:

$$
\begin{aligned}
& \left(f_{1}\right)_{i, j}^{(n+1)}={ }_{y} \bar{f}_{1}^{(n)}-\frac{\epsilon^{2}\left[g_{i, j}+\left(\left(f_{2}\right)_{x y}\right)_{i, j}^{(n)}\right]}{2}, \\
& \left(f_{2}\right)_{i, j}^{(n+1)}={ }_{x} \bar{f}_{2}^{(n)}-\frac{\epsilon^{2}\left[h_{i, j}+\left(\left(f_{1}\right)_{x y}\right)_{i, j}^{(n)}\right]}{2},
\end{aligned}
$$

where

$$
\begin{aligned}
{ }_{y} \bar{f}_{1} & =\frac{\left(f_{1}\right)_{i+1, j}+\left(f_{1}\right)_{i-1, j}}{2}, \\
{ }_{x} \bar{f}_{2} & =\frac{\left(f_{2}\right)_{i, j+1}+\left(f_{2}\right)_{i, j-1}}{2},
\end{aligned}
$$

and $n$ is the iteration number.

Similarly, we rewrite (19) as

$$
\nabla^{2} p=l(x, y, t)
$$

and

$$
\nabla^{2} p_{i, j}=4 \frac{\bar{p}_{i, j}-p_{i, j}}{\epsilon^{2}}
$$

where

$$
\bar{p}_{i, j}=\frac{p_{i+1, j}+p_{i-1, j}+p_{i, j+1}+p_{i, j-1}}{4} .
$$

We then suggest the following iterative procedure:

$$
p_{i, j}^{(n+1)}=\bar{p}_{i, j}^{(n)}-\frac{\epsilon^{2} l_{i, j}}{4},
$$

where $n$ is the iteration number.

\section{APPENDIX D}

\section{Computing Ground Truth and MEF Velocities and Pressures for Synthetic Image Sequences}

The following algorithm is followed for computing the ground truth flow field in Fig. 3:

\section{- $\quad$ Step 1}

Use $\rho^{(1)}$ and $\rho^{(2)}$ along with (8) and (9) to find $\left(\bar{u}^{(1)}, \bar{v}^{(1)}\right)$. We will assume this to be our ground-truth flow, $\left(\bar{u}_{\mathrm{gt}}^{(1)}, \bar{v}_{\mathrm{gt}}^{(1)}\right)$. Superscripts indicate time steps.

- $\quad$ Step 2

Use $\rho^{(2)}$ and $\rho^{(3)}$ along with (8) and (9) to find $\left(\bar{u}_{\mathrm{gt}}^{(2)}, \bar{v}_{\mathrm{gt}}^{(2)}\right)$.

- Step 3 Use (1), $\left(\bar{u}_{\mathrm{gt}}^{(1)}, \bar{v}_{\mathrm{gt}}^{(1)}\right)$, and $\rho^{(1)}$ to compute $\rho^{*(2)}$. Similarly, use $\left(\bar{u}_{\mathrm{gt}}^{(2)}, \bar{v}_{\mathrm{gt}}^{(2)}\right)$ and $\rho^{*(2)}$ to compute $\rho^{*(3)}$.

\section{- $\quad$ Step 4}

Compute MEF flow rates, $\left(\bar{u}_{\mathrm{MEF}}^{(1)}, \bar{v}_{\mathrm{MEF}}^{(1)}\right)$ and $\left(\bar{u}_{\mathrm{MEF}}^{(2)}, \bar{v}_{\mathrm{MEF}}^{(2)}\right)$, using density pairs $\left(\rho^{(1)}, \rho^{*(2)}\right)$ and $\left(\rho^{*(2)}, \rho^{*(3)}\right)$, respectively, and (8) and (9).

- $\quad$ Step 5

Use $\left(\bar{u}_{\mathrm{gt}}^{(1)}, \bar{v}_{\mathrm{gt}}^{(1)}\right)$ and $\left(\bar{u}_{\mathrm{gt}}^{(2)}, \bar{v}_{\mathrm{gt}}^{(2)}\right)$ in (11) and (12) to compute the ground-truth pressure. Assume that there is no external forcing.

- $\quad$ Step 6

Use $\left(\bar{u}_{\mathrm{MEF}}^{(1)}, \bar{v}_{\mathrm{MEF}}^{(1)}\right)$ and $\left(\bar{u}_{\mathrm{MEF}}^{(2)}, \bar{v}_{\mathrm{MEF}}^{(2)}\right)$ in (11) and (12) to compute the MEF pressure. Assume that there is no external forcing.

\section{ACKNOWLEDGMENTS}

This research was supported by the US Office of Naval Research, the Alfred P. Sloan Foundation, the US National Oceanographic Partnership Program, and is a contribution to the Census of Marine Life. The authors thank Margrit Betke for her useful discussions and her suggestion to apply MEF to the problem of cell division.

\section{REFERENCES}

[1] A. Amini, "A Scalar Function Formulation for Optical Flow," Proc. European Conf. Computer Vision, pp. 125-131, 1994.

[2] P. Anandan, "A Computational Framework and an Algorithm for the Measurement of Visual Motion," Int'l J. Computer Vision, vol. 2, pp. 283-310, 1989. 
[3] M. Andrews, Z. Gong, and P. Ratilal, "High Resolution Population Density Imaging of Random Scatterers with the Matched Filtered Scattered Field Variance," The J. Acoustical Soc. of Am., vol. 126 , no. 3, pp. 1057-1068, 2009 .

[4] J. Barron, D. Fleet, and S. Beauchemin, "Performance of Optical Flow Techniques," Int'l J. Computer Vision, vol. 12, no. 1, pp. 43-77, 1994.

[5] G.K. Batchelor, An Introduction to Fluid Dynamics. Cambridge Univ. Press, 1967.

[6] R. Battiti, E. Amaldi, and C. Koch, "Computing Optical Flow across Multiple Scales: An Adaptive Coarse-to-Fine Strategy," Int'l J. Computer Vision, vol. 6, no. 2, pp. 133-145, June 1991.

[7] D. Béréziat, I. Herlin, and L. Younes, "A Generalized Optical Flow Constraint and Its Physical Interpretation," Proc. IEEE Conf. Computer Vision and Pattern Recognition, vol. 2, pp. 487-492, 2000.

[8] D. Béréziat and J.-P. Berroir, "Motion Estimation on Meteorological Infrared Data Using a Total Brightness Invariance Hypothesis," Environmental Modeling and Software, vol. 15, pp. 513-519, 2000.

[9] T. Corpetti, D. Heitz, G. Arroyo, E. Mémin, and A. Santa-Cruz, "Fluid Experimental Flow Estimation Based on an Optical-Flow Scheme," Experiments in Fluids, vol. 40, pp. 80-97, 2006.

[10] I.D. Couzin and J. Krause, "Self-Organization and Collective Behavior in Vertebrates," Advances in the Study of Behavior, vol. 32, pp. 1-75, 2003.

[11] G. Danuser and C.M. Waterman-Storer, "Quantitative Fluorescent Speckle Microscopy: Where It Came from and Where It Is Going," J. Microscopy, vol. 211, pp. 191-207, Sept. 2003.

[12] W.J. Devenport, M.C. Rife, S.I. Liapis, and G.J. Follin, "The Structure and Development of a Wing-Tip Vortex," J. Fluid Mechanics, vol. 312, pp. 67-106, 1996

[13] V. Devlaminck and J.-P. Dubus, "Estimation of Compressible or Incompressible Deformable Motions for Density Images," Proc. Int'l Conf. Image Processing, vol. 1, pp. 125-128, Sept. 1996.

[14] L.G. Ekatarina, M.I. Maxim, I.A. Fazly, and J.R. McIntosh, "Force Production by Disassembling Microtubules," Nature, vol. 438, pp. 384-388, 2005.

[15] W. Enkelmann, "Investigation of Multigrid Algorithms for the Estimation of Optical Flow Fields in Image Sequences," Computer Vision, Graphics, and Image Processing, vol. 43, no. 2, pp. 150-177, Aug. 1988.

[16] D.M. Farmer, M.V. Trevorrow, and Q.B. Pederson, "Intermediate Range Fish Detection with a $12-\mathrm{kHz}$ Sidescan Sonar," The J. Acoustical Soc. of Am., vol. 106, pp. 2481-2490, 1999.

[17] J. Fitzpatrick, "A Method for Calculating Fluid Flow in Time Dependent Images Based on the Continuity Equation," Proc. IEEE Conf. Computer Vision and Pattern Recognition, pp. 78-81, 1985.

[18] D.J. Fleet and A.D. Jepson, "Computation of Component Image Velocity from Local Phase Information," Int'l J. Computer Vision, vol. 5, pp. 77-104, 1990.

[19] S. Freeman, "Cell Division," Biological Science, Prentice Hall, 2002.

[20] Z. Gong, M. Andrews, S. Jagannathan, R. Patel, J.M. Jech, N.C. Makris, and P. Ratilal, "Low-Frequency Target Strength and Abundance of Shoaling Atlantic Herring (Clupea Harengus) in the Gulf of Maine during the Ocean Acoustic Waveguide Remote Sensing 2006 Experiment," The J. Acoustical Soc. of Am., vol. 127, pp. 104-123, 2010.

[21] T. Guerrero et al., "Dynamic Ventilation Imaging from FourDimensional Computed Tomography," Physics in Medicine and Biology, vol. 51, no. 4, pp. 777-791, 2006.

[22] D.J. Heeger, "Optical Flow Using Spatiotemporal Filters," Int'l J. Computer Vision, vol. 1, pp. 279-302, 1988.

[23] B. Horn and B. Schunck, "Determining Optical Flow," Artificial Intelligence, vol. 17, pp. 185-203, 1981

[24] I. Huse and E. Ona, "Tilt Angle Distribution and Swimming Speed of Overwintering Norwegian Spring Spawning Herring," Int'l Council for the Exploration of the Sea J. Marine Science, vol. 53, pp. 863-873, 1996.

[25] S. Inoue and H. Sato, "Cell Motility by Labile Association of Molecules: The Nature of Mitotic Spindle Fibers and Their Role in Chromosome Movement," The J. General Physiology, vol. 50, pp. 259-292, 1967.

[26] S. Inoue and E.D. Salmon, "Force Generation by Microtubule Assembly/Disassembly in Mitosis and Related Movements," Molecular Biology of the Cell, vol. 6, no. 12, pp. 1619-1640, 1995.
[27] S. Jagannathan, I. Bertsatos, D. Symonds, H.T. Nia, A. Jain, M. Andrews, Z. Gong, R. Nero, L. Ngor, M. Jech, O.R. Godø, S. Lee, P. Ratilal, and N. Makris, "Ocean Acoustic Waveguide Remote Sensing (OAWRS) of Marine Ecosystems," Marine Ecology Progress Series, vol. 395, 2009.

[28] K.E. Kasza, A.C. Rowat, J. Liu, T.E. Angelini, C.P. Brangwynne, G.H. Koenderink, and D.E. Weitz, "The Cell as a Material," Current Opinion in Cell Biology, vol. 19, pp. 101-107, 2007.

[29] L.D. Landau and E.M. Lifshitz, Mechanics. Pergammon Press, 1976.

[30] H. Lodish, A. Berk, P. Matsudaira, C.A. Kaiser, M. Krieger, M.P. Scott, S.L. Zipursky, and J. Darnell, Molecular Cell Biology. Freeman and Company, 2004.

[31] B. Lucas and T. Kanade, "An Iterative Image Registration Technique with an Application to Stereo Vision," Proc. Defense Advanced Research Projects Agency Image Understanding Workshop, pp. 121-130, 1981

[32] N.C. Makris, P. Ratilal, D. Symonds, S. Jagannathan, S. Lee, and R. Nero, "Fish Population and Behavior Revealed by Instantaneous Continental Shelf Scale Imaging," Science, vol. 311, pp. 660-663, 2006.

[33] N.C. Makris, P. Ratilal, S. Jagannathan, Z. Gong, M. Andrews, I. Bertsatos, O.R. Godø, R. Nero, and M. Jech, "Critical Population Density Triggers Rapid Formation of Vast Oceanic Fish Shoals," Science, vol. 323, no. 5922, pp. 1734-1737, 2009.

[34] P.L.M. de Maupertuis, "Accord de Diffrentes Lois de la Nature Qui Avaient Jusqu'ici paru Incompatibles" Mm. As. Sc. Paris, p. $417,1744$.

[35] D. Metaxas and D. Terzopoulos, "Shape and Nonrigid Motion and Structure," IEEE Trans. Pattern Analysis and Machine Intelligence, vol. 15 , no. 6, pp. 580-591, June 1993.

[36] O.A. Misund, A. Fernö, T. Pitcher, and B. Totland, "Tracking Herring Schools with a High Resolution Sonar. Variations in Horizontal Area and Relative Echo Intensity," Int'l Council for the Exploration of the Sea J. Marine Science, vol. 55, pp. 58-66, 1998.

[37] H.-H. Nagel, "On the Estimation of Optical Flow: Relations between Different Approaches and Some New Results," Artifical Intelligence, vol. 33, pp. 299-324, 1987.

[38] Y. Nakajima, H. Inomatad, H. Nogawab, Y. Satoa, S. Tamuraa, K. Okazakic, and S. Torii, "Physics-Based Flow Estimation of Fluids," Pattern Recognition, vol. 36, no. 5, pp. 1203-1212, 2003.

[39] R. Ortega, G. Devés, and A. Carmona, "Bio-Metals Imaging and Speciation in Cells Using Proton and Synchrotron Radiation X-Ray Microspectroscopy," J. Royal Soc. Interface, vol. 6, pp. S649S658, 2009

[40] T. Potapova and G. Gorbsky, "Classic Mitosis in a Vertebrate Cell," ASCB Image and Video Library, VID-31, http://cellimages. ascb.org/u?/p4041coll12,233, Aug. 2007.

[41] A. Pentland and B. Horowitz, "Recovery of Nonrigid Motion and Structure," IEEE Trans. Pattern Analysis and Machine Intelligence, vol. 13, no. 7, pp. 730-742, July 1991.

[42] T.J. Pitcher and J. Parrish, The Behavior of Teleost Fishes, T. J. Pitcher ed., pp. 363-439. Chapman and Hall, 1993.

[43] J.L. Prince, "Motion Estimation from Tagged MR Image Sequences," IEEE Trans. Medical Imaging, vol. 11, no. 2, pp. 238-249, June 1992.

[44] K. Rhode et al., "Validation of an Optical Flow Algorithm to Measure Blood Flow Waveforms in Arteries Using Dynamic Digital X-Ray Images," Proc. SPIE Conf., p. 1414, 2000.

[45] P.G. Saffman, M.J. Ablowitz, E. Hinch, J.R. Ockendon, and P.J Olver, Vortex Dynamics. Cambridge Univ. Press, 1992.

[46] J. Simpson and J. Gobat, "Robust Velocity Estimates, Stream Functions and Simulated Lagrangian Drifters from Sequential Spacecraft Data," IEEE Trans. Geosciences and Remote Sensing, vol. 32, no. 3, pp. 479-493, May 1994.

[47] A. Singh, "An Estimation-Theoretic Framework for Image Flow Computation," Proc. Third IEEE Int'l Conf. Computer Vision, pp. 168-177, 1990.

[48] S.M. Song and R.M. Leahy, "Computation of 3D Velocity Fields from 3D Cine CT Images of a Human Heart," IEEE Trans. Medical Imaging, vol. 10, no. 3, pp. 295-306, Sept. 1991.

[49] D. Suter, "Motion Estimation and Vector Splines," Proc. IEEE CS Conf. Computer Vision and Pattern Recognition, pp. 939-942, June 1994.

[50] D. Terzopoulos and K. Waters, "Analysis and Synthesis of Facial Image Sequences Using Physical and Anatomical Processes," IEEE Trans. Pattern Analysis and Machine Intelligence, vol. 15, no. 6 pp. 569-579, June 1993 
[51] J. Toner and Y. Tu, "Long-Range Order in a Two-Dimensional Dynamical XY Model: How Birds Fly Together," Physical Rev. Letters, vol. 75, no. 23, pp. 4326-4329, 1995.

[52] J. Toner and Y. Tu, "Flocks, Herds, and Schools: A Quantitative Theory of Flocking," Physical Rev. E, vol. 58, pp. 4828-4858, 1998.

[53] S. Uras, F. Girosi, A. Verri, and V. Torre, "A Computational Approach to Motion Perception," Biological Cybernetics, vol. 60, pp. 79-87, 1988.

[54] A. Vlasenko and C. Schnörr, "Variational Approaches to Image Fluid Flow Estimation with Physical Priors" Imaging Measurement Methods for Flow Analysis, pp. 247-256, Springer, 2009.

[55] A.M. Waxman, J. Wu, and F. Bergholm, "Convected Activation Profiles and Receptive Fields for Real Time Measurement of Short Range Visual Motion," Proc. IEEE Conf. Computer Vision and Pattern Recognition, pp. 723-771, 1988.

[56] R.P. Wildes et al. "Recovering Estimates of Fluid Flow from Image Sequence Data," Computer Vision and Image Understanding, vol. 80, pp. 246-266, 2000.

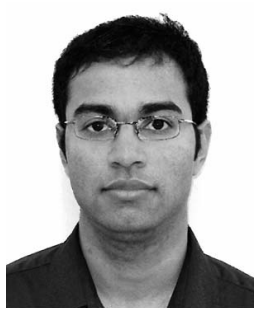

Srinivasan Jagannathan received the BTech degree in ocean engineering from the Indian Institute of Technology (IIT) Madras, India, in 2004. Currently, he is working toward the $\mathrm{PhD}$ degree in mechanical engineering at the Massachusetts Institute of Technology (MIT). He is a research assistant at the Laboratory for Undersea Remote Sensing at MIT. He is the recipient of the US Office of Naval Research Graduate Fellowship in Underwater Acoustics.

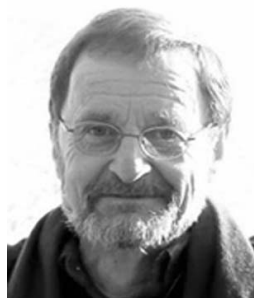

Berthold Klaus Paul Horn received the BScEng degree from the University of the Witwatersrand in 1965, and the SM and PhD degrees from the Massachusetts Institute of Technology (MIT) in 1968 and 1970, respectively. $\mathrm{He}$ is a professor of electrical engineering and computer science at MIT. He is the author or coauthor of three books on machine vision and programming. He was awarded the Rank Prize for pioneering work leading to practical vision systems in 1989 and was elected a fellow of the American Association of Artificial Intelligence in 1990. He was elected to the National Academy of Engineering in 2002.

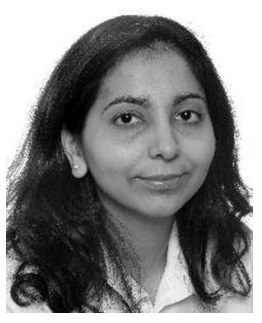

Purnima Ratilal received the PhD degree in acoustics from the Massachusetts Institute of Technology (MIT) in 2002. She is an associate professor of electrical and computer engineering at Northeastern University, Boston. She was previously a postdoctoral associate in the Department of Ocean Engineering at MIT (2002-2004) and a research scientist at Singapore's DSO National Laboratories (19941998). She has extensive experimental and theoretical experience in remote sensing with acoustics and ultrasonics. She was awarded the US Office of Naval Research (ONR) Postdoctoral Award in Ocean Acoustics in 2002, the Bruce Lindsay Award by the Acoustical Society of America in 2006, the ONR Young Investigator Award in 2007, and the Presidential Early Career Award for Scientists and Engineers in 2008.

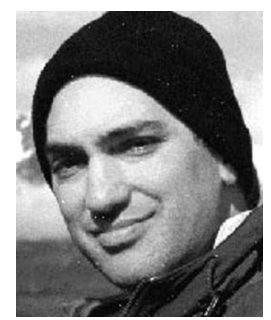

Nicholas Constantine Makris is a professor at the Massachusetts Institute of Technology. For more information, see http:// acoustics.mit.edu/ faculty/makris/makris.html.

$>$ For more information on this or any other computing topic, please visit our Digital Library at www.computer.org/publications/dlib. 